\title{
On the renormalized volume of hyperbolic 3-manifolds
}

\author{
Kirill Krasnov** and Jean-Marc Schlenker ${ }^{\dagger}$
}

November 2006 (v3)

\begin{abstract}
The renormalized volume of hyperbolic manifolds is a quantity motivated by the AdS/CFT correspondence of string theory and computed via a certain regularization procedure. The main aim of the present paper is to elucidate its geometrical meaning. We use another regularization procedure based on surfaces equidistant to a given convex surface $\partial N$. The renormalized volume computed via this procedure is equal to what we call the $W$-volume of the convex region $N$ given by the usual volume of $N$ minus the quarter of the integral of the mean curvature over $\partial N$. The $W$-volume satisfies some remarkable properties. First, this quantity is self-dual in the sense explained in the paper. Second, it verifies some simple variational formulas analogous to the classical geometrical Schläfli identities. These variational formulas are invariant under a certain transformation that replaces the data at $\partial N$ by those at infinity of $M$. We use the variational formulas in terms of the data at infinity to give a simple geometrical proof of results of Takhtajan et al on the Kähler potential on various moduli spaces.
\end{abstract}

\section{Introduction}

The renormalized volume. In this paper we study the so-called renormalized volume of hyperbolic 3manifolds whose definition is motivated by the AdS/CFT correspondence of string theory Wit98. In the context of this correspondence one is interested in computing the gravity action

$$
S_{g r}[g]=\frac{1}{2} \int_{M}(R-2 \Lambda) d v+\int_{\partial M} H d a
$$

for an Einstein metric $g$ on d-dimensional manifold $M$. Here we have set the dimensionful Newton constant typically present in the action (11) to $8 \pi G=1$, and $\Lambda$ is the cosmological constant, which is assumed to be negative. The quantity $R$ is the curvature scalar, $H$ is the mean curvature of the boundary $\partial M$, and $d v, d a$ are the volume and area forms correspondingly.

In the context of AdS/CFT correspondence the manifold $M$ is non-compact with a conformal boundary $\partial M$. The metric $g$ on $M$ determines the conformal class of the boundary $\partial M$. One would like to compute $S_{g r}$ as a functional of this conformal class. However, because the manifold $M$ is non-compact the functional $S_{g r}[g]$ diverges. One notices, however, that this divergence is of a special type. Thus, let us introduce a compact sub-manifold $N \subset M$ and compute the action $S_{g r}[g, N]$ (1) inside $N$. As one sends $\partial N$ towards the infinity of $M$ one can show the divergent part of $S_{g r}[g, N]$ is given by an integral over $\partial N$ of a local quantity expressible in terms of the first and second fundamental forms of $\partial N$. This suggests an idea of renormalization in which these divergent quantities are subtracted, after which the limit $\partial N \rightarrow \partial M$ can be taken. This idea works in any dimension, see [BK99 for the required subtraction procedure.

\footnotetext{
* School of Mathematical Sciences, University of Nottingham, Nottingham, NG7 2RD, UK

†Laboratoire Emile Picard, UMR CNRS 5580, Institut de Mathématiques, Université Paul Sabatier, 31062 Toulouse Cedex 9, France. http://www.picard.ups-tlse.fr/ schlenker.
} 
3-dimensional manifolds. In this paper we are interested in the simplest case of 3-dimensional spaces. The simplifications arising in this case are as follows. First, Einstein equations in 3 dimensions imply the metric $g$ to be of constant curvature. Thus, in our case of a negative cosmological constant we are led to consider constant negative curvature manifolds. In 3 dimensions the radius of curvature $l$ is related to the cosmological constant in a simple way $l=1 / \sqrt{-\Lambda}$. We shall set the radius of curvature $l=1$ in what follows, which in 3 dimensions is equivalent to $\Lambda=-1$. Thus, the Riemannian manifolds $(M, g)$ we are going to consider are hyperbolic. As is well known from Bers' work Ber60] on simultaneous uniformization and from its generalization to arbitrary Kleinian manifolds, the manifolds $(M, g)$ are completely characterized by the conformal structures of all the boundary components of $M$. We would like to compute the gravity action $S_{g r}$ as a functional of the conformal structure of $\partial M$. The constant curvature condition gives $R=-6$. Therefore, the gravity action reduces to

$$
S_{g r}=-2 \int_{M} d v+\int_{\partial M} H d a .
$$

It is now easy to show that the volume $V(N), N \subset M$ diverges as $(1 / 2) A(\partial N)$, where $A(\partial N)$ is the area of the boundary of $N$. There is also the so-called logarithmic divergence to be discussed below. The integrated mean curvature diverges as $\int_{\partial N} H d a \sim 2 A(\partial N)$. Altogether we see that the gravity action functional diverges as $A(\partial N)$. It seems natural, therefore, to introduce a renormalized gravity action given by

$$
S_{g r}[g]=\frac{1}{2} \int_{M}(R-2 \Lambda) d v+\int_{\partial M}(H-1) d a .
$$

This action can then be computed for any compact sub-manifold $N \subset M$. However, because of the logarithmic divergence present in the volume, the limit $N \rightarrow M$ does not exist. It is this logarithmic divergence that causes all the difficulties that we are now to discuss.

In an even number of dimensions, when a more involved but similar in principle subtraction procedure is used, there is no logarithmic divergence and the limit can indeed be shown to exist and to be independent on how exactly the surfaces $\partial N$ are taken to approach the conformal boundary $\partial M$. However, when the dimension is odd, as is in the case of interest for us, the limit of (3) does not exist, except in the special situation when all the boundary components are of genus one. In general, the volume of $N$ grows as the logarithm of the area of $\partial N$ times the Euler characteristic of $\partial N$. As we shall see below, one can subtract this divergence as well, but the resulting renormalized gravity action (or volume) then turns out to depend on the limiting procedure. This is why in odd dimensions the renormalized gravity action fails to be a true invariant of $M$. It is precisely for this reason that the concept of renormalized volume has not been developed by the geometry community. Indeed, the fact that the volume of a hyperbolic manifold grows as half of the area of its boundary has been known for a very long time and is mentioned, for example, in Thurston's famous notes on the subject. It was also realized however that the limit $V(N)-(1 / 2) A(\partial N)$ does not exist. The geometry community instead concentrated on e.g. a canonically defined volume of the convex core of $M$. The logarithmic divergence (referred to as the conformal anomaly in the physics literature) is studied in more details in HS98.

In Kra00] one of us studied the renormalized volume of Schottky 3-manifolds. It was shown that there is a way to define the limit of the quantity $V(N)-(1 / 2) A(\partial N)$ by choosing a foliation of $M$ near its boundary by a family of surfaces $S_{\rho}[\phi]$ parameterized by a "Liouville" (real-valued) field on the conformal boundary of $M$. Once such a family of surfaces is used, the limiting procedure becomes well-defined. Indeed, one takes the quantity $V_{\rho}-(1 / 2) A_{\rho}$ and subtracts from it $2 \pi \rho(g-1)$, where $g$ is the genus of the boundary of $M$. It is then easy to see that the limit exists and defines the renormalized volume $V_{R}(M, \phi)$, which in addition to being a function of the conformal structure of $\partial M$ also depends on the Liouville field $\phi$ on $\partial M$. It was moreover shown by an explicit computation that $V_{R}(M, \phi)$ is equal to the Liouville action $S_{L}(\partial M, \phi)$ of Takhtajan and Zograf TZ87. To get a "canonical" quantity that depends only on the conformal structure of $\partial M$ one can evaluate $V_{R}(M, \phi)=S_{L}(\partial M, \phi)$ on the canonical Liouville field corresponding to the metric of constant curvature -1 in the conformal class of $\partial M$. This canonical $\phi$ can also be obtained by extremizing the functional $V_{R}(M, \phi)$ keeping the area of $\partial M$ as determined by $\phi$ fixed. This variational principle leads to the unique canonically defined $\phi$. One gets the "canonical" renormalized volume that depends only on the conformal structure of $\partial M$.

This way of defining the limit can be generalized to an arbitrary Kleinian manifold. This has been done in [TT03, where the limiting procedure of [Kra00] was also improved in the sense that an invariant family of 
Epstein surfaces Eps84 was used for regularization, see also an earlier paper by one of the authors Kra01, where the same Epstein family of surfaces was used for regularization, but of the CS formulation of gravity instead. In all cases, the renormalized volume obtained via the limiting procedure was shown to agree with the Liouville action on $\partial M$. Variation of the renormalized volume $=$ Liouville action under the changes of the conformal structure of $\partial M$ were studied in [TZ87, TT03]. It was shown that in all the cases the renormalized volume is equal to the Kähler potential for the Weil-Petersson metric on the moduli space of $\partial M$. For quasiFuchsian spaces this implies, e.g. the quasi-Fuchsian reciprocity of McMullen McM00.

The renormalized volume and equidistant foliations. In the present paper we undertake a further study of the renormalized volume of hyperbolic 3-manifolds. We show that the limiting procedure via which the volume is defined can be somewhat de-mystified by considering for regularization a family of surfaces equidistant to a given one, following an idea already used by C. Epstein [PP01] (and more recently put to use in [KS05]). Thus, the main idea of the present work is to obtain the renormalized volume by taking a convex domain $N \subset M$, and compute the renormalized volume of $M$ with respect to $N$ as

$$
V_{R}(M, N)=V(N)+\lim _{\rho \rightarrow \infty}\left(V\left(\partial N, \partial N_{\rho}\right)-(1 / 2) A\left(\partial N_{\rho}\right)-\sum_{i} 2 \pi \rho\left(g_{i}-1\right)\right),
$$

where $V\left(\partial N, \partial N_{\rho}\right)$ is the volume between the boundary $\partial N$ of the domain $N$ and the surface $\partial N_{\rho}$ located a distance $\rho$ from $\partial N$. The quantity $A\left(\partial N_{\rho}\right)$ is the area of the surface $\partial N_{\rho}$, the sum in the last term is taken over all boundary components of $M$ and $g_{i}$ are the genera of these boundary components. The convexity of the domain $N$ ensures that the equidistant surfaces $\partial N_{\rho}$ exist all the way to infinity. This ensures that the limit $\rho \rightarrow \infty$ can be taken. Similarly, using the combination (2) and subtracting the area of $\partial N_{\rho}$, as well as the term linear in $\rho$, one defines the renormalized gravity action. In both cases the limit exists and can be computed in terms of the volume or the gravity action for $N$, see below for the corresponding expressions.

The limiting procedure used in Kra01, TT03] is an example of the limiting procedure described above, for the Epstein surfaces Eps84 are equidistant. Thus, the renormalized volume of references [Kra00, [TT03] is an example of the renormalized volume (4) where $N$ is a compact domain of $M$ contained inside a particular Epstein surface. However, it is obvious that the renormalized volume (4) is more general as the domain $N$ in (4) can be an arbitrary convex domain. It is also clear that the renormalized volume defined via (4) is "the most general one". Indeed, the only constraint that enters into the definition (44) is that equidistant surfaces are used for regularization. However, this seems to be a necessary requirement to be able to subtract the logarithmic divergence. Thus, there does not seem any other way to define the renormalized volume.

Renormalized volume as the $W$-volume. The starting point is a simple formula for the renormalized volume (4):

$$
V_{R}(M, N)=W(N)-\sum_{i} \pi\left(g_{i}-1\right)
$$

where the sum in the last term is taken over all the boundary components. Here the $\mathrm{W}$-volume is defined as

$$
W(N):=V(N)-\frac{1}{4} \int_{\partial N} H d a .
$$

This formula for $V_{R}$ is a special case of a formula found by C. Epstein [PP01] for the renormalized volume of hyperbolic manifolds in any dimension.

Thus, the renormalized volume of $M$ with respect to $N$ is, apart from an uninteresting term given by a multiple of the Euler characteristic of the boundary, just the $\mathrm{W}$-volume of the domain $N$. All our other results concern this $\mathrm{W}$-volume. Note already that $W(N)$ is not equal to the Hilbert-Einstein functional of $N$ with its usual boundary term, it differs from it in the coefficient of the boundary term. 
Variation formula. We prove a formula for the first variation of $\mathrm{W}$ under changes of the metric inside $N$. This formula is a simple consequence of the formula obtained by Igor Rivin and one of us in [RS99]. It reads

$$
\delta W(N)=\frac{1}{4} \int_{\partial N}\left\langle\delta I I-\frac{H}{2} \delta I, I\right\rangle d a .
$$

Thus, this formula suggest that the $W$-volume of a domain $N$ is a complicated functional of the shape of this domain, as well as of the manifold $M$. However, as we shall show, this functional depends on all these data in a very specific way, through a certain combination that we introduce below and refer to as the metric "at infinity".

Self-duality. One of the most interesting properties of the $\mathrm{W}$-volume is that it is self-dual. Thus, we recall that the Einstein-Hilbert functional

$$
I_{E H}(N):=V(N)-\frac{1}{2} \int_{\partial N} H d a
$$

for a compact domain $N \subset H^{3}$ of the hyperbolic space (note a different numerical factor in front of the second term) is nothing but the dual volume. Thus, recall that there is a duality between objects in $H^{3}$ and objects in $d S_{3}$, the $2+1$ dimensional de Sitter space. Under this duality geodesic planes in $H^{3}$ are dual to points in $d S_{3}$, etc. This duality between domains in the two spaces is easiest to visualize for convex polyhedra (see [RH93]), but the duality works for general domains as well. The fact that (8) is the volume of the dual domain then is a simple consequence of the Schläfli formula, analogous to (7), see the main text below.

Thus, we can write:

$$
{ }^{*} V(N)=V\left({ }^{*} N\right)=V(N)-\frac{1}{2} \int_{\partial N} H d a
$$

for the volume of the dual domain. This immediately shows that

$$
W(N)=V(N)-\frac{1}{4} \int_{\partial N} H d a=\frac{V(N)+{ }^{*} V(N)}{2} .
$$

Thus, the $W$-volume is self-dual in that this quantity for $N$ is equal to this quantity for the dual domain ${ }^{*} N$ : $W(N)=W\left({ }^{*} N\right)$. In the main text we shall also verify the self-duality more directly by applying the Legendre transform to $W(N)$.

The $W$-volume and the Chern-Simons formulation. An interesting remark is that there is a very simple expression for the $W$-volume in terms of the so-called Chern-Simons formulation of $2+1$ gravity Wit89. In this formulation the gravity action in the so-called first order formalism, in which the independent variables are the triads and the spin connection, is shown to be given by the difference of two Chern-Simons actions. This is easily shown for the "bulk" term of the gravity action, while to get the boundary term as in (11) one needs in addition a certain set of boundary terms in terms of the Chern-Simons connections. Remarkably, the combination that plays this role in the $W$-volume, namely

$$
\frac{1}{2} \int_{M}(R-2 \Lambda)+\frac{1}{2} \int_{\partial M} K
$$

which is different from the usual Einstein-Hilbert action, is exactly what appears naturally in the Chern-Simons formulation, without the need for any boundary terms. We refer the reader to e.g. Kra01 for a demonstration of this fact, see formula (3.7) of this reference as well as the related discussion. It would be of interest to understand the relation, if any, between the self-duality of the $W$-volume and the fact that it has such a simple expression in the Chern-Simons formulation. 
Description "from infinity". The other important property of the W-volume is that it can be interpreted as a quantity that depends on the metric "at infinity" only, instead of being a functional of the "shape" of the boundary $\partial N$ of $N \subset M$. To demonstrate this one introduces the first and second fundamental forms $I^{*}, I^{*}$ obtained from those on the surfaces $\partial N_{\rho}$ as $\rho \rightarrow \infty$ by a simple rescaling, see the main text. The relation between the data at infinity and those on $\partial N$ are as follows

$$
I^{*}=\frac{1}{2}(I+I I) I^{-1}(I+I I), \quad I^{*}=\frac{1}{2}(I+I I) I^{-1}(I-I I) .
$$

These relations can be inverted, with the inverse relations looking exactly the same, with starred quantities replaced by non-starred everywhere, see (32) below. We note that the metric $I^{*}$ is in the conformal class of the boundary $\partial M$, but its precise form of course depends on the convex domain $N$ used.

The new variation formula. The formula (7) can then be re-written in terms of the variations $\delta I^{*}, \delta I^{*}$, the result being

$$
\delta W(N)=-\frac{1}{4} \int_{\partial N}\left\langle\delta I^{*}-\frac{H^{*}}{2} \delta I^{*}, I^{*}\right\rangle d a^{*} .
$$

This formula could be compared to a similar one given by Anderson in dimension 4 And01.

Thus, the variational formula for the $W$-volume is essentially invariant under the transformation (12). One simple corollary of this formula is that the extremum of the $W$-volume under variations of the metric $I^{*}$ that keep the area of $\partial M$ as defined by $I^{*}$ fixed occurs for the hyperbolic (i.e. constant negative curvature) $I^{*}$. As there is always the unique canonical such $I^{*}$ (of given area), the extremal $W$-volume becomes truly a functional of the conformal structure of the boundary components of $M$. By considering the second variation, we also prove that this extremum of $W$ is a maximum.

It is important to note that the second fundamental form $I^{*}$ at infinity is completely determined by $I^{*}$ on all the boundary component. This is essentially a consequence of Bers' simultaneous uniformization. We give a direct proof of this fact in the main text. This fact implies that $W(N)$ is a functional of $I^{*}$ only, a very important property of the renormalized volume. If one wishes, one can obtain a more canonical functional that depends on $M$ only, by taking the metric $I^{*}$ to be the canonical metric of curvature -1 in the conformal class of infinity of $M$. It is this "extremal" $W$-volume, called $W_{M}$ in the latter sections here, that is of most interest due to the following.

An important immediate corollary of (13) is the theorem by Takhtajan and Teo [TT03 that the extremal renormalized volume is equal to the Kähler potential on the moduli space of Kleinian manifolds. We refer to the main text for a proof of this. We note that our proof is entirely geometrical and avoids a reasonably complicated cohomology machinery that is necessary in TT03. For this reason our proof can be immediately extended even to situations where the methods of [TT03] are inapplicable, such as manifolds with cone singularities. See more remarks on this case below.

Positivity. The (extremal, i.e the one for the hyperbolic $I^{*}$ ) W-volume coincides with a multiple of the potential studied by Teo in [Teo05. The result of this reference implies that the extremal W-volume is a nonnegative function on the moduli space of manifolds, attaining the zero value only on the Fuchsian manifolds. Here we obtain a similar result for the $W$-volume of the convex core $C_{M}$ of $M$, see section 4 . These positivity results for the $W$-volume of two different convex domains in $M$ lead us to suggest that the $W$-volume of any convex domain might be positive. We do not attempt to answer this question in the present work leaving it for future research.

Manifolds with particles and the Teichmüller theory of surfaces with cone singularities. One key feature of the arguments presented in this work is that they are always local, in the sense that they depend on local quantities defined on the boundaries of compact subsets of quasi-Fuchsian manifolds. Thus, we make only a very limited use of the fact that the quasi-Fuchsian manifolds are actually quotients of hyperbolic 3-space by a group of isometries. One place where this is used is in the proof of the fact that $I^{*}$ is determined by $I^{*}$ (actually 
a direct consequence of the Bers double uniformization theorem). We expect that all the results should extend from quasi-Fuchsian (more generally geometrically finite) manifolds to the "quasi-Fuchsian manifolds with particles" which were studied e.g. in KS05, MS06. Those are actually cone-manifolds, with cone singularities along infinite lines running from one connected component of the boundary at infinity to the other, along which the cone angle is less than $\pi$.

One problem towards such an extension is that although in (non-singular) quasi-Fuchsian setting the Bers double uniformization theorem shows that everything is determined by the conformal structure at infinity, there is as yet no such result in the corresponding case "with particles". It appears likely, however, that such a statements holds for "quasi-Fuchsian manifolds with particles"; a first step towards it is made in [MS06, while the second step is one of the objects of a work in progress between the second author and C. Lecuire.

The result of [MS06] could actually already be used - even without a global Bers type theorem for hyperbolic manifolds with particles - to obtain results on the Teichmüller-type space of hyperbolic metrics with $n$ cone singularities of prescribed angles on a closed surface of genus $g$. Note that this space, which can be denoted by $\mathcal{T}_{g, n, \theta}$ (with $\theta=\left(\theta_{1}, \cdots, \theta_{n}\right) \in(0, \pi)^{n}$ ) is topologically the same as the "usual" Teichmüller space $\mathcal{T}_{g, n}$ of hyperbolic metrics with $n$ cusps (with a one-to-one correspondence from [Tro91]) but it has a natural "WeilPetersson" metric which is different. It might follow from the considerations made here, extended to quasiFuchsian manifolds with particles, that this "Weil-Petersson" metric is still Kähler, with the renormalized volume playing the role of a Kähler potential. A global Bers-type theorem would not be necessary for this because, given any hyperbolic metric $h \in \mathcal{T}_{g, n, \theta}$ on a surface $\Sigma$, we can consider the "Fuchsian" hyperbolic manifold with particles defined as the warped product

$$
M:=\left(\Sigma \times \mathbb{R}, d t^{2}+\cosh (t)^{2} h\right) .
$$

Clearly the conformal structure at infinity on both connected components of the boundary at infinity of $M$ are given by $h$. Moreover it follows from [MS06] that if $h_{-}:=h$ and $h_{+}$is in a small neighborhood $U \subset \mathcal{T}_{g, n, \theta}$ of $h$ then there exists a unique quasi-Fuchsian manifold with particles, close to $M$, with conformal structures at infinity given by $h_{-}$and $h_{+}$. The arguments developed here (extended to this singular context) should show that the renormalized volume is a Kähler potential for the natural Weil-Petersson metric on $\mathcal{T}_{g, n, \theta}$ restricted to $U$. We leave such an extension to quasi-Fuchsian cone manifolds to future work.

Acknowledgment. We would like to thank Rafe Mazzeo for useful comments on a previous version of this text, and in particular for pointing out reference [PP01].

\section{Preliminaries}

In this section we collect various background materials useful further on in the paper.

Extrinsic invariants of surfaces in $H^{3}$. Let $S$ be a smooth surface in $H^{3}$. Its Weingarten (or shape) operator is a bundle morphism $B: T S \rightarrow T S$ defined by:

$$
B x:=-\nabla_{x} N,
$$

where $N$ is the unit normal vector field to $S$ and $\nabla$ is the Levi-Cività connection of $H^{3}$. B is then self-adjoint with respect to the induced metric on $S$, which we call $I$ here. The second fundamental form of $S$ is then defined by:

$$
I I(x, y):=I(B x, y)=I(x, B y),
$$

for any two vectors $x$ and $y$ tangent to $S$ at the same point. The third fundamental form of $S$ is defined as:

$$
\text { III }(x, y):=I(B x, B y) .
$$

When $B$ has no zero eigenvalue, $I I I$ is a Riemannian metric on $S$. 
The Gauss and Codazzi equations. The Weingarten operator satisfies two equations on $S$, the Codazzi equation:

$$
d^{\nabla} B=0,
$$

and the Gauss equation:

$$
\operatorname{det}(B)=K+1
$$

where $K$ is the curvature of the induced metric $I$ on $S$. $\operatorname{det}(B)$ is called the extrinsic curvature of $S$, denoted by $K_{e}$.

The Gauss and Codazzi equation are the only relations satisfied by the first and second fundamental forms of a surface. This can be expressed in a mildly complicated way as the "fundamental theorem of surface theory" stated below. Here and elsewhere we use a fairly natural convention: given two bilinear symmetric forms $g$ and $h$ on $S$, with $g$ positive definite, we denote by $g^{-1} h$ the unique bundle morphism $b: T S \rightarrow T S$, self-adjoint for $g$, such that $h(x, y)=g(b x, y)$ for any two vectors $x, y$ tangent to $S$ at the same point. For instance, $I^{-1} I I=B$ by definition.

Theorem 2.1. Let $g$ and $h$ be two smooth symmetric bilinear forms on a simply connected surface $S$, with $g$ positive definite at each point. Define $b:=g^{-1} h, h=\operatorname{Tr}(b)$, and $k_{e}=\operatorname{det}(B)$. If $g, h$ satisfy the constraints:

$$
\begin{array}{cc}
\nabla^{g} h=0 & \text { (Codazzi) } \\
k_{e}=K_{g}+1 & \text { (Gauss), }
\end{array}
$$

where $K_{g}$ is the Gauss curvature of $g$, then there exists a unique immersion of $S$ into the hyperbolic space $H^{3}$ such that $g, h$ are the induced metric and second fundamental forms of $S$ respectively.

The next lemma, which is elementary and well-known, describes the behavior of the surfaces at constant distance from a fixed surface.

Lemma 2.2. Let $S$ be a surface in $H^{3}$, with bounded principal curvatures, and let $I, B$ be the first fundamental form and the shape operator of $S$ correspondingly. Let $S_{\rho}$ be the surface at distance $\rho$ from $S_{\rho}$. Then, for sufficiently small $\rho$ the induced metric on $S_{\rho}$ is:

$$
I_{\rho}(x, y)=I((\cosh (\rho) E+\sinh (\rho) B) x,(\cosh (\rho) E+\sinh (\rho) B) y) .
$$

Here $E$ is the identity operator.

Note that this lemma also holds for a surface $S$ in any hyperbolic 3-manifold $M$, not necessarily $H^{3}$. We also note that when the surface $S$ is convex, then the expression (16) gives the induced metric on any surface $\rho>0$, where $\rho$ increases in the convex direction. A proof of this lemma, and of the two corollaries which follow, can be found in KS05.

Corollary 2.3. The area of the surfaces $S_{\rho}$ is given by:

$$
\begin{aligned}
A(\rho) & =\int_{S} \operatorname{det}(\cosh (\rho) E+\sinh (\rho) B) d a \\
& =\int_{S}\left(\cosh ^{2}(\rho)+\cosh (\rho) \sinh (\rho) H+\sinh ^{2}(\rho) K_{e}\right) d a .
\end{aligned}
$$

Corollary 2.4. The integrated mean curvature of $S_{\rho}$ is given by:

$$
\int_{S_{\rho}} H d a=\frac{\partial}{\partial \rho} A(\rho)=\int_{S}\left(\sinh (2 \rho)\left(1+K_{e}\right)+\cosh (2 \rho) H\right) d a .
$$




\section{The new volume}

Definition. We define the $W$-volume of a compact hyperbolic manifold with boundary as follows.

Definition 3.1. Let $M$ be a hyperbolic 3-manifold, and $N$ be a compact subset of $M$ with boundary $\partial N$. Then:

$$
W(N):=V(N)-\frac{l^{2}}{4} \int_{\partial N} H d a,
$$

where $V(N)$ is the volume of $N, l$ is the radius of curvature of the space, $H$ is the mean curvature of the boundary, and da is the area element of the metric induced on $\partial N$.

Note that the volume defined above does not coincide with the usual Einstein-Hilbert action:

$$
S_{E H}[g]=\int_{M} \sqrt{g}(R-2 \Lambda)+2 \int_{\partial M} H
$$

evaluated on a metric of constant curvature. Indeed, for such a metric $R-2 \Lambda=4 \Lambda$. Thus, defining the radius of curvature as $\Lambda=-1 / l^{2}$ we have:

$$
I_{E H}(N):=-\frac{l^{2}}{4} S_{E H}(N)=V(N)-\frac{l^{2}}{2} \int_{\partial N} H,
$$

which is different from (20). We will set the radius of curvature $l$ to one in what follows.

The following property of $W(N)$ is obvious:

Lemma 3.2. The $W$-volume is additive: if $N_{1}$ and $N_{2}$ are two compact sub-manifolds of $M$ such that $N_{1} \cap N_{2}$ is a disjoint union of connected components of the boundary of both $N_{1}$ and $N_{2}$ then:

$$
W\left(N_{1} \cup N_{2}\right)=W\left(N_{1}\right)+W\left(N_{2}\right) .
$$

Proof. This is obvious when $N_{1}, N_{2}$ do not share any boundary components. For $N_{1}, N_{2}$ such that a part of their respective boundaries is shared the additivity follows from the fact that the mean curvatures of that boundary component have the opposite sign as viewed from $N_{1}$ and $N_{2}$.

We note that the on-shell Einstein-Hilbert action (21) is also additive. Thus, at this stage there is no reason to prefer (20) to (21). However, as we shall see in the next section, it is the quantity (20) that behaves much more regularly for non-compact hyperbolic manifolds as well as for compact ones. Also, as we shall presently see, it is the $W$-volume that is the self-dual one.

Self-duality. Here we prove self-duality of the new $W$-volume by considering its Legendre transform. We will need to use the variation formula (77) given in the introduction. An immediate consequence of this formula is that the variation of the $W(N)$ under the condition that the "conjugate" momentum

$$
\pi=-\frac{1}{4}\left(I I-\frac{H}{2} I\right)
$$

is fixed is given by

$$
\delta W(N)=\int_{\partial N}\langle\pi, \delta I\rangle .
$$

The quantity (22) is exactly the unique combination of $I$, II such that when it is kept fixed the variation of $W(N)$ produces exactly $\pi$. The dual $\mathrm{W}$-volume can now be obtained by a Legendre transform:

$$
{ }^{*} W(N):=-\int_{\partial N}\langle\pi, I\rangle+W(N) .
$$


We see that, because $\pi$ is traceless, the $W$-volume is self-dual: ${ }^{*} W(N)=W(N)$. Note that a similar argument applied to the usual volume $V(N)$ of a domain $N$ shows that its Legendre transform is given by the EinsteinHilbert functional $I_{E H}(N)$. This demonstrates the fact that the Einstein-Hilbert functional is the dual volume $I_{E H}(N)={ }^{*} V(N)$ to which we referred to in the introduction.

We have so far discussed the duality only in the context of a compact domain $N \subset H^{3}$. The duality is, however, more general and holds also for domains in more general hyperbolic 3-manifolds. Note, however, that in this more general context one has to be careful about the geometrical meaning of the dual volume. Indeed, the 3-manifold dual to $M$ is modeled on $d S_{3}$ spacetime, and typically has two disconnected components, each having an internal boundary - a surface dual to the boundary of the convex core in $M$. Given a convex domain $N$ that contains the convex core $C_{M}$ one can meaningfully talk about the dual domain in $d S_{3}$ as being a domain in the dual manifold located between the surfaces dual to $\partial N$ and the internal boundary of ${ }^{*} M$. This discussion serves as a good introduction to the following section in which we discuss precisely those more general hyperbolic 3 -manifolds for which the notion of the $W$-volume is of interest.

\section{Convex co-compact hyperbolic manifolds}

Definitions and first properties. We first need to define convex co-compact hyperbolic manifolds.

Definition 4.1. A complete hyperbolic 3-manifold $M$ is convex co-compact if there is a compact subset $N \subset M$ whose boundary $\partial N$ is convex and such that the normal exponential map from $\partial N$ to the conformal boundary $\partial M$ is a homeomorphism. Each connected component of the complement $M \backslash N$ is called a hyperbolic end of $M$.

Note that the condition on $N$ is equivalent to the fact that $N$ is strongly convex in the sense that any geodesic segment in $M$ with endpoints in $N$ is actually contained in $N$.

Simple examples of convex co-compact manifolds are: Schottky manifolds, each having one hyperbolic end; quasi-Fuchsian manifolds with two hyperbolic ends.

The new volume $W(N)$ is especially interesting because it is (almost) defined not only for compact subsets $N$ as in the above definition, but also for the hyperbolic ends. The following computation is central to motivate the definition that follows.

Lemma 4.2. Consider a hyperbolic end of a convex co-compact manifold $M$, and let $S$ be a connected component of the boundary $\partial N$ of the compact subset $N$ of the definition 4.1 . The $W$-volume of the sub-manifold contained between the surfaces $S$ and $S_{\rho}$ is given by:

$$
W\left[S, S_{\rho}\right]=-\frac{\rho}{2} \int_{S} K d a=2 \pi \rho(g-1),
$$

where $g$ is the genus of $S$.

Proof. We have:

$$
\begin{aligned}
V(\rho) & =\int_{0}^{\rho} A(r) d r \\
& =\int_{0}^{\rho} \int_{S} \operatorname{det}(\cosh (r) E+\sinh (r) B) d a d r \\
& =\int_{0}^{\rho} \int_{S} \cosh ^{2}(r)+H \cosh (r) \sinh (r)+K_{e} \sinh ^{2}(r) d a d r \\
& =\int_{0}^{\rho} \int_{S}\left(\cosh ^{2}(r)+\sinh ^{2}(r)\right)+K \sinh ^{2}(r)+H \cosh (r) \sinh (r) d a d r \\
& =\int_{0}^{\rho} \int_{S} \cosh (2 r)+K \frac{\cosh (2 r)-1}{2}+H \frac{\sinh (2 r)}{2} d a d r
\end{aligned}
$$

so that:

$$
V(\rho)=\frac{1}{2} \int_{S}\left(\sinh (2 \rho)+\frac{K}{2}(\sinh (2 \rho)-2 \rho)+\frac{H}{2}(\cosh (2 \rho)-1)\right) d a .
$$


Now the $W$-volume is given by:

$$
V(\rho)-\frac{1}{4} \int_{S_{\rho}} H d a+\frac{1}{4} \int_{S} H d a .
$$

The formula (25) follows by combining (26) with (19).

The relative $W$-volume. Thus, the $W$-volume of a portion of a hyperbolic end is just the thickness of the portion considered times $2 \pi(g-1)$. This fact motivates the following definition:

Definition 4.3. Let $M$ be a convex co-compact hyperbolic 3-manifold with one or more hyperbolic ends. Let $N$ be a compact convex subset of $M$ as in the definition 4.1. The $W$-volume $W(M, N)$ of $M$ relative to $N$ is defined as the $W$-volume of $N$.

Note that $W(N)$ is the same as the $W$-volume of $M$ with the $W$-volumes (25) of the hyperbolic ends removed. Thus, one could also refer to the volume $W(M, N)$ as the renormalized volume. Indeed, it has a close relation to the renormalized volume that has appeared in the literature. Here we would like to give a comparison to the renormalized volume.

Definition 4.4. Let $M$ be a convex co-compact hyperbolic 3-manifold, and let $S_{r}^{i}$ be a foliation by equidistant surfaces near each component $i$ of the boundary. The renormalized volume of $M$ relative to foliations $S_{\rho}^{i}$ is defined as

$$
V_{R}\left(M, S_{\rho}\right):=\lim _{\rho \rightarrow \infty} V(\rho)-\sum_{i} \frac{1}{2} A_{\rho}^{i}-\sum_{i} 2 \pi \rho_{i}\left(g_{i}-1\right),
$$

Here $V(\rho)$ is the volume of the subset of $M$ bounded by surfaces $S_{\rho}^{i}, A_{\rho}^{i}$ and $g_{i}$ are the areas and genera of the surfaces $S_{\rho}^{i}$ correspondingly. The limit is a multiple limit of all $\rho_{i} \rightarrow \infty$.

When $M$ is convex co-compact there is a natural foliation of each end by surfaces equidistant to the (strongly) convex subset $N$. In this case we will talk about the renormalized volume $V_{R}(M, N)$ of $M$ relative to the (strongly) convex subset $N$.

Lemma 4.5. Let $M$ be convex co-compact, and let $N$ be a (strongly) convex subset of $M$ (as in the definition 4.1). The renormalized volume of $M$ relative to $N$ is the $W$-volume minus a multiple of the Euler characteristic of the boundary:

$$
V_{R}(M, N)=W(M, N)-\sum_{i} \pi\left(g_{i}-1\right) .
$$

The sum is taken over the boundary components.

This formula is the 3-dimensional case of a formula given by C. Epstein for the renormalized volume of hyperbolic manifolds in PP01. We include a proof for the reader's convenience.

Proof. Consider one of the hyperbolic ends. Let $S$ be the corresponding boundary component of $\partial N$. The area (17) of $S_{\rho}$ can be rewritten as:

$$
A(\rho)=\int_{S}\left(\cosh (2 \rho)+\frac{H}{2} \sinh (2 \rho)+\frac{K}{2}(\cosh (2 \rho)-1)\right) d a .
$$

Subtracting half of this from (26) we get:

$$
V(\rho)-A(\rho) / 2-2 \pi \rho(g-1)=-\frac{1}{4} e^{-2 \rho} \int_{S}(2-H+K) d a-\frac{1}{4} \int_{S} H d a-\pi(g-1) .
$$

The result now follows by taking the limit $\rho \rightarrow \infty$ and adding the terms corresponding to all the different ends. 
Thus, the result (27) shows that the renormalized volume relative to a strongly convex subset $N$ is basically the volume (20) we have defined, apart from a constant term proportional to the Euler characteristic of $\partial N$. However, as it is clear from the proof, the two quantities agree only after the limit is taken. For a finite $\rho$ the renormalized volume is a complicated functional, and it is only in the limit that a simplification occurs. The volume (20) we have defined is in contrast simple even for a finite $\rho$, as well as for any compact domain $N$. One could try to define an analog of the renormalized volume for a general subset $N \subset M$ by taking the volume minus half of the area of $\partial N$. This functional however fails to be additive and is thus of a very limited interest, apart from the limiting case when the surface $\partial N$ is sent to infinity. All this makes it clear that the functional $W(N)$ is much more natural to consider than the one that plays a role in the definition of the renormalized volume.

Having motivated and defined the $W$-volume, the natural question to ask is what this quantity depends on. From its definition one may expect that it depends on the shape of the convex subset $N$ in $M$ in a complicated way. However, as we shall see, this dependence is actually rather simple in that the $W$-volume is just a certain functional of the so-called "asymptotic" metric constructed using the fundamental forms of the boundary of $N$. We deal with this in the next section. However, before we study this question, let us demonstrate certain positivity properties of the $W$-volume.

Positivity estimates on $W$. We note that some of the quantities considered here are always positive on the convex core of a quasi-Fuchsian hyperbolic manifold. We actually prove this here under a technical hypothesis which is conjecturally always satisfied.

Lemma 4.6. Let $M$ be a quasi-Fuchsian manifold, and let $C_{M}$ be its convex core. Suppose that $C_{M}$ is the Gromov-Hausdorff limit of a sequence of convex cores of hyperbolic manifolds with bending laminations along closed curves. Then $I_{E H}\left(C_{M}\right) \geq 0$, with equality exactly when $M$ is Fuchsian.

A short explanation on the hypothesis is needed. Given $M$, let $\lambda$ be the measured bending lamination on the boundary of its convex core. It is known (see [BO04, Lec06]) that $\lambda$ is the limit of the measured bending laminations of a sequence of quasi-Fuchsian manifolds $M_{n}$ for which the convex core converge, in the Gromov-Hausdorff distance, to the convex core of a quasi-Fuchsian manifold $M^{\prime}$, and that the measured bending lamination on the boundary of the convex core of $M^{\prime}$ is $\lambda$. According to a conjecture of Thurston, this last point should imply that $M^{\prime}=M$, and then the hypothesis made in the lemma would be useless.

Proof. We consider in the proof that $M$ is not Fuchsian, since in that case it is quite obvious that $I_{E H}\left(C_{M}\right)=0$.

Suppose first that the bending lamination of $C_{M}$ is along disjoint closed curves. Let $l_{i}$ and $\lambda_{i}$ be the lengths and bending angles at those closed curves. It is then known BO04 that there exists a one-parameter family of quasi-Fuchsian manifolds, $M_{t}, 0 \leq t \leq 1$, with $M_{0}$ Fuchsian, $M_{1}=M$, and such that, for all $t \in[0,1]$, the bending lamination of the convex core of $M_{t}$ is $t$ times the measured bending lamination of the convex core of $M$. In other terms, the bending angle of the curve $i$ on the boundary of the convex core of $M_{t}$ is $t \lambda_{i}$. Let $l_{i}(t)$ be the length of this curve.

Now a simple computation using the Schläfli formula shows that:

$$
\frac{d I_{E H}\left(C_{M_{t}}\right)}{d t}=-\frac{1}{2} \sum_{i} t \lambda_{i} \frac{d l_{i}(t)}{d t}
$$

However Choi and Series [CS06] have recently proved that, in this context, the matrix of the differential of the lengths with respects to the angles is negative definite. Since the $\theta_{i}(t)=t \lambda_{i}$ here, it follows that:

$$
\sum_{i} \lambda_{i} \frac{d l_{i}(t)}{d t}<0
$$

and therefore $I_{E H}\left(C_{M_{t}}\right)$ is a strictly increasing function of $t$. Since it vanishes for $t=0$, it follows that $I_{E H}(M)>0$.

If the bending lamination of $M$ is general - i.e., it is not supported on closed curves - the result can be obtained, thanks to the technical hypothesis in the lemma, by approximating $M$ by quasi-Fuchsian manifolds for which the bending lamination of the boundary of the convex core is along closed curves. 
Corollary 4.7. Under the same hypothesis, $W\left(C_{M}\right) \geq 0$, with equality exactly when $M$ is Fuchsian.

Proof. This immediately follows from the previous lemma since

$$
W(M)=I_{E H}(M)+\frac{1}{4} \int_{\partial M} H .
$$

These results should be compared to a recent result by Teo Teo05 that shows that the $W$-volume extremized in a certain way, to be explained below, is positive. This other extremized volume is simply the volume of a different convex domain in $M$. Taken together, these results suggest that the $W$-volume of any convex domain $N$ might be positive. We will not attempt to prove this statement in the present work.

\section{Description "from infinity"}

The metric at infinity. In this section we switch from a description of the renormalized volume from the boundary of a convex subset to the boundary at infinity of $M$. This description from infinity is remarkably similar to the previous one from the boundary of a convex subset.

Lemma 5.1. Let $M$ be a convex co-compact hyperbolic 3-manifold, and let $N \subset M$ be compact and "strongly" convex with smooth boundary. Let $S_{\rho}$ be the equidistant surfaces from $\partial N$. The induced metric on $S_{\rho}$ is asymptotic, as $\rho \rightarrow \infty$, to $(1 / 2) e^{2 \rho} I^{*}$, where $I^{*}=(1 / 2)(I+2 I I+I I)$ is defined on $\partial N$.

Proof. Follows from Lemma 2.2.

It is the metric $I^{*}$ that will play such a central role in what follows, so we would like to state some of its properties.

Lemma 5.2. The curvature of $I^{*}$ is

$$
K^{*}:=\frac{2 K}{1+H+K_{e}} .
$$

Proof. The Levi-Cività connection of $I^{*}$ is given, in terms of the Levi-Cività connection $\nabla$ of $I$, by:

$$
\nabla_{x}^{*} y=(E+B)^{-1} \nabla_{x}((E+B) y) .
$$

This follows from checking the 3 points in the definition of the Levi-Cività connection of a metric:

- $\nabla^{*}$ is a connection.

- $\nabla^{*}$ is compatible with $I^{*}$.

- it is torsion-free (this follows from the fact that $E+B$ verifies the Codazzi equation: $\left(\nabla_{x}(E+B)\right) y=$ $\left.\left(\nabla_{y}(E+B)\right) x\right)$.

Let $\left(e_{1}, e_{2}\right)$ be an orthonormal moving frame on $S$ for $I$, and let $\beta$ be its connection 1-form, i.e.:

$$
\nabla_{x} e_{1}=\beta(x) e_{2}, \nabla_{x} e_{2}=-\beta(x) e_{1} .
$$

Then the curvature of $I$ is defined as: $d \beta=-K d a$.

Now let $\left(e_{1}^{*}, e_{2}^{*}\right):=\sqrt{2}\left((E+B)^{-1} e_{1},(E+B)^{-1} e_{2}\right)$; clearly it is an orthonormal moving frame for $I^{*}$. Moreover the expression of $\nabla^{*}$ above shows that its connection 1 -form is also $\beta$. It follows that $K d a=-d \beta=K^{*} d a^{*}$, so that:

$$
K^{*}=K \frac{d a}{d a^{*}}=\frac{K}{(1 / 2) \operatorname{det}(E+B)}=\frac{2 K}{1+H+K_{e}} .
$$


We note that the metric $I^{*}$ is defined for any surface $S \subset M$. However, it might have singularities (even when the surface $S$ is smooth) unless $S$ is strictly horospherically convex, i.e., its principal curvatures are less than 1 (which implies that it remains on the concave side of the tangent horosphere at each point). If $S$ is a strictly horospherically convex surface $S$ embedded in a hyperbolic end of $M$ then the metric $I^{*}$ is guaranteed to be the in the conformal class of the (conformal) boundary at infinity of $M$. For a general surface $S$ the "asymptotic" metric has nothing to do with the conformal infinity, and in particular, does not have to be in the conformal class of the boundary.

The $W$-volume as a functional of $I^{*}$. We claim that the $W$-volume of a convex co-compact manifold $M$ relative to a convex subset $N$ is a functional of only the metric $I^{*}$ that is built using the fundamental forms of the boundary $\partial N$. This claim can be substantiated in several ways. One way is to refer to the results about the renormalized volume. It is known from [Kra00, TT03] that the renormalized volume of $M$ is given by the so-called Liouville functional for the asymptotic metric $I^{*}$. To prove this result one uses an explicit foliation of the covering space $H^{3}$ by certain equidistant surfaces. The easy part of the computation is then to identify the "bulk" part of the Liouville action. The hard part is to show that all the boundary terms that arise are exactly the boundary of the fundamental domain terms necessary to define the Liouville action. As we have said, this computation is done in the covering space and is not particularly illuminating as far as the geometry of the problem is concerned. In this paper we would like to give a more geometric perspective. We demonstrate the above assertion by proving an explicit variational formula for $W(N)$ in terms of $I^{*}$. However, before we do this, we would like to introduce some other quantities defined "at infinity".

Second fundamental form at infinity. We have already defined the metric "at infinity". Let us now add to this a definition of what can be called the second fundamental form at infinity.

Definition 5.3. Given a surface $S$ with the first, second and third fundamental forms I, II and III, we define the first and second fundamental forms "at infinity" as:

$$
\begin{aligned}
I^{*}=\frac{1}{2}(I+2 I I+I I I) & =\frac{1}{2}(I+I I) I^{-1}(I+I I)=\frac{1}{2} I((E+B) \cdot,(E+B) \cdot), \\
I^{*}=\frac{1}{2}(I-I I I) & =\frac{1}{2}(I+I I) I^{-1}(I-I I)=\frac{1}{2} I((E+B) \cdot,(E-B) \cdot) .
\end{aligned}
$$

It is then natural to define:

$$
B^{*}:=\left(I^{*}\right)^{-1} I^{*}=(E+B)^{-1}(E-B),
$$

and

$$
\text { III }^{*}:=I^{*}\left(B^{*} \cdot, B^{*} \cdot\right)=I((E-B) \cdot,(E-B) \cdot) .
$$

Note that, for a surface which has principal curvatures strictly bounded between -1 and $1, I I^{*}$ is also a smooth metric and its conformal class corresponds to that on the other component of the boundary at infinity. This is a simple consequence of the Lemma 2.2 and the fact that when the principal curvatures are strictly bounded between $-1,1$ the foliation by surfaces equidistant to $S$ extends all the way through the manifold $M$. Such manifolds were called almost-Fuchsian in our work [KS05.

As before, those definitions make sense for any surface, but it is only for a convex surface (or more generally for a horospherically convex surface) that the fundamental forms so introduced are guaranteed to have something to do with the actual conformal infinity of the space.

The Gauss and Codazzi equations at infinity. We also define $H^{*}:=\operatorname{tr}\left(B^{*}\right)$. The Gauss equation for "usual" surfaces in $H^{3}$ is replaced by a slightly twisted version.

Remark 5.4. $H^{*}=-K^{*}$ : the mean curvature at infinity is equal to minus the curvature of $I^{*}$. 
Proof. By definition, $H^{*}=\operatorname{tr}\left((E+B)^{-1}(E-B)\right)$. An elementary computation (for instance based on the eigenvalues of $B$ ) shows that

$$
H^{*}=\frac{2-2 \operatorname{det}(B)}{1+\operatorname{tr}(B)+\operatorname{det}(B)} .
$$

But we have seen (as Equation (29) ) that $K^{*}=2 K /\left(1+H+K_{e}\right.$ ), the result follows because, by the Gauss equation, $K=-1+\operatorname{det}(B)$.

However, the "usual" Codazzi equation holds at infinity.

Remark 5.5. $d^{\nabla^{*}} B^{*}=0$.

Proof. Let $u, v$ be vector fields on $\partial_{\infty} M$. Then it follows from the expression of $\nabla^{*}$ found above that:

$$
\begin{aligned}
\left(d^{\nabla^{*}} B^{*}\right)(x, y) & =\nabla_{x}^{*}\left(B^{*} y\right)-\nabla_{y}^{*}\left(B^{*} x\right)-B^{*}[x, y] \\
& =(E+B)^{-1} \nabla_{x}\left((E+B) B^{*} y\right)-(E+B)^{-1} \nabla_{y}\left((E+B) B^{*} x\right)-B^{*}[x, y] \\
& =(E+B)^{-1} \nabla_{x}((E-B) y)-(E+B)^{-1} \nabla_{y}((E-B) x)-(E+B)^{-1}(E-B)[x, y] \\
& =(E+B)^{-1}\left(d^{\nabla}(E-B)\right)(x, y) \\
& =0 .
\end{aligned}
$$

Inverse transformations. The transformation $I, I I \rightarrow I^{*}, I^{*}$ is invertible. The inverse is given explicitly by:

Lemma 5.6. Given $I^{*}, I^{*}$ the fundamental forms I, II such that (30) holds are obtained as:

$$
\begin{aligned}
& \left.I=\frac{1}{2}\left(I^{*}+I^{*}\right)\left(I^{*}\right)^{-1}\left(I^{*}+I^{*}\right)=\frac{1}{2} I^{*}\left(\left(E+B^{*}\right) \cdot,\left(E+B^{*}\right) \cdot\right)\right), \\
& \left.I I=\frac{1}{2}\left(I^{*}+I^{*}\right)\left(I^{*}\right)^{-1}\left(I^{*}-I^{*}\right)=\frac{1}{2} I^{*}\left(\left(E+B^{*}\right) \cdot,\left(E-B^{*}\right) \cdot\right)\right) .
\end{aligned}
$$

Moreover,

$$
B=\left(E+B^{*}\right)^{-1}\left(E-B^{*}\right) .
$$

Having an expression for the fundamental forms of a surface in terms of the one at infinity one can re-write the metric of Lemma 2.2 induced on surfaces equidistant to $S$ in terms of $I^{*}, I^{*}$.

Lemma 5.7. The metric (16) induced on the surfaces equidistant to $S$ can be re-written in terms of the fundamental forms "at infinity" as:

$$
I_{\rho}=\frac{1}{2} e^{2 \rho} I^{*}+I^{*}+\frac{1}{2} e^{-2 \rho} \Pi^{*} .
$$

This lemma shows the significance of $I^{*}$ as being the constant term of the metric. This lemma also shows clearly that when the equidistant foliation extends all the way through $M$ (i.e. when the principal curvatures on $S$ are in $(-1,1))$, the conformal structure at the second boundary component of $M$ is that of $I I^{*}=I^{*}\left(I^{*}\right)^{-1} I^{*}$. Thus, in this particular case of almost-Fuchsian manifolds, the knowledge of $I^{*}$ on both boundary components of $M$ is equivalent to the knowledge of $I^{*}, I^{*}$ near either component. In other words, $I^{*}$ is determined by $I^{*}$. This statement is more general and works for manifolds other than almost-Fuchsian. 
Fundamental Theorem of surface theory "from infinity". Let us now recall that the Fundamental Theorem of surface theory, Theorem 2.1. states that given $I$, II on $S$ there is a unique embedding of $S$ into the hyperbolic space. Then (16) gives an expression for the metric on equidistant surfaces to $S$, and thus describes a hyperbolic manifold $M$ in which $S$ is embedded, in some neighborhood of $S$. It would be possible to state a similar result for hyperbolic ends, uniquely determined by $I^{*}$ and $I^{*}$ at infinity. But there is also an analogous theorem, based on a classical result of Bers [Ber60, in which the first (and only the first) form at infinity is used. This can be compared with arguments used in Sch02.

Theorem 5.8. Given a convex co-compact 3-manifold $M$, and a metric $I^{*}$ (on all the boundary components of $M)$ in the conformal class of the boundary, there is a unique foliation of each end of $M$ by convex equidistant surfaces $S_{\rho} \subset M(1 / 2)\left(I_{\rho}+2 I_{\rho}+I I I_{\rho}\right)=e^{2 \rho} I^{*}$, where $I_{\rho}, I_{\rho}, I I I_{\rho}$ are the fundamental forms of $S_{\rho}$.

Remark 5.9. Note that one does not need to specify $I^{*}$. The first fundamental form $I^{*}$ (but on all the boundary components) is sufficient.

Proof. The surfaces in question can be given explicitly as an embedding of the universal cover $\tilde{S}$ of $S$ into the hyperbolic space. Thus, let $(\xi, y), \xi>0, y \in C$ be the usual upper half-space model coordinates of $H^{3}$. Let us write the metric at infinity as

$$
I^{*}=e^{\phi}|d z|^{2},
$$

where $\phi$ is the Liouville field covariant under the action of the Kleinian group giving $M$ on $S^{2}$. The surfaces are given by the following set of maps: $E p s_{\rho}: S^{2} \rightarrow H^{3}, z \rightarrow(\xi, y)$ (here Eps stands for Epstein, who described these surfaces in Eps84):

$$
\begin{array}{r}
\xi=\frac{\sqrt{2} e^{-\rho} e^{-\phi / 2}}{1+(1 / 2) e^{-2 \rho} e^{-\phi}\left|\phi_{z}\right|^{2}}, \\
y=z+\phi_{\bar{z}} \frac{e^{-2 \rho} e^{-\phi}}{1+(1 / 2) e^{-2 \rho} e^{-\phi}\left|\phi_{z}\right|^{2}} .
\end{array}
$$

As is shown by an explicit computation, the metric induced on the surfaces $S_{\rho}$ is given by (34) with

$$
\begin{array}{r}
I^{*}=\frac{1}{2}\left(\theta d z^{2}+\bar{\theta} d \bar{z}^{2}\right)+\phi_{z \bar{z}} d z d \bar{z}, \\
\theta=\phi_{z z}-\frac{1}{2}\left(\phi_{z}\right)^{2} .
\end{array}
$$

Thus, we see that $I^{*}$ is determined by the conformal factor in (35).

Remark 5.10. This theorem itself implies that the renormalized volume only depends on $I^{*}$. Indeed, the foliation $S_{\rho}$ of the ends does depend only on $I^{*}$, and this foliation can be used for regularization and subtraction procedure. Then the fact that the $W$-volume is essentially the renormalized volume implies that $W$-volume is a functional of $I^{*}$ only. In the next section we will find a formula for the first variation of this functional.

Corollary 5.11. If the principal curvatures at infinity (eigenvalues of $B^{*}$ ) are positive the map Eps $\rho_{\rho}$ is a homeomorphism onto its image for any $\rho$.

Proof. We first note that the map Eps $\rho$ is not always a homeomorphism, and the surfaces $S_{\rho}$ are not necessarily convex, but for sufficiently large $\rho$ both things are true. A condition that guarantees that $E p s_{\rho}$ is a homeomorphism for any $\rho$ is stated above. This condition can be obtained from the requirement that the principal curvatures of surfaces $S_{\rho}$ are in $[-1,1]$. Let us consider the surface $S:=S_{\rho=0}$ the first and second fundamental forms of which are given by (32) (this immediately follows from (34)). The shape operator of this surface is then given by $B=\left(E+B^{*}\right)^{-1}\left(E-B^{*}\right)$. It is then clear that the principal curvatures of $S$ are given by $k_{i}=\left(1-k_{i}^{*}\right) /\left(1+k_{i}^{*}\right)$, where the $k_{i}^{*}$ are the "principal curvatures" (eigenvalues) of $B^{*}$. The latter are easily shown to be given by

$$
k_{1,2}^{*}=e^{-\phi}\left(\phi_{z \bar{z}} \pm \sqrt{\theta \bar{\theta}}\right)
$$


It is now easy to see that the condition $k_{1,2} \in(-1,1)$ is equivalent to the condition $k_{1,2}^{*}>0$. This is a necessary and sufficient condition for the foliation by surfaces $S_{\rho}$ to extend throughout $M$. If this condition is satisfied the map Eps $\rho$ is a homeomorphism for any $\rho$.

Interestingly, this condition makes sense not only in the quasi-Fuchsian situation but is more general. Thus, for example, it applies to the Schottky manifolds. But for the Schottky manifolds with their single boundary component the foliation by equidistant surfaces $S_{\rho}$ cannot be smooth for arbitrary $\rho$. It is clear that surfaces must develop singularities for some value of $\rho$. We therefore get a very interesting corollary:

Corollary 5.12. There is no Liouville field $\phi$ on $\mathbb{C}$ invariant under a Schottky group such that $\phi_{z \bar{z}}$ is greater than $\left|\phi_{z z}-(1 / 2) \phi_{z}^{2}\right|$ everywhere on $\mathbb{C}$.

Proof. Indeed, if such a Liouville field existed, we could have used it to construct a smooth equidistant foliation for arbitrary values of $\rho$, but this is impossible.

Similar statement holds for a Kleinian group with more than two components of the domain of the discontinuity.

\section{The Schläfli formula "from infinity"}

In this section we obtain a formula for the first variation of the renormalized volume. The computation of this section is a bit technical. Readers not interested in the details are advised to skip this section on the first reading. The result of the computation is given by the formula (45) below.

The Schläfli formula. As we have seen in the previous sections, the renormalized volume of a convex cocompact hyperbolic 3-manifold $M$ can be expressed as the $\mathrm{W}$-volume of any convex domain $N \subset M$. The W-volume is equal to the volume of $N$ minus the quarter of the integral of the mean curvature over the boundary of $N$. Let us consider what happens if one changes the metric in $M$. As was shown in RS99, the following formula for the variation of the volume holds

$$
2 \delta V(N)=\int_{\partial N}\left(\delta H+\frac{1}{2}\langle\delta I, I I\rangle\right) d a .
$$

Here $H$ is the trace of the shape operator $B=I^{-1} I I$, and the expression $\langle A, B\rangle \operatorname{stands}$ for $\operatorname{tr}\left(I^{-1} A I^{-1} B\right)$. We can use this to get the following expression for the variation of the $\mathrm{W}$-volume:

$$
\delta W(N)=\frac{1}{2} \int_{\partial N}\left(\delta H+\frac{1}{2}\langle\delta I, I I\rangle\right) d a-\frac{1}{4} \int_{\partial N} \delta H d a-\frac{1}{4} \int_{\partial N} H \delta(d a)
$$

so that

$$
\delta W(N)=\frac{1}{4} \int_{\partial N}\left(\delta H+\left\langle\delta I, I I-\frac{H}{2} I\right\rangle\right) d a .
$$

To get the last equality we have used the obvious equality

$$
d a^{\prime}=\frac{1}{2} \operatorname{tr}\left(I^{-1} \delta I\right)=\frac{1}{2}\langle\delta I, I\rangle d a .
$$

The formula (41) can be further modified using

$$
\delta H=\delta\left(\operatorname{tr}\left(I^{-1} I I\right)\right)=-\operatorname{tr}\left(I^{-1}(\delta I) I^{-1} I I\right)+\operatorname{tr}\left(I^{-1} \delta I I\right)=-\langle\delta I, I I\rangle+\langle I, \delta I I\rangle .
$$

We get

$$
\delta W(N)=\frac{1}{4} \int_{\partial N}\left\langle\delta I-\frac{H}{2} \delta I, I\right\rangle d a .
$$

It is this formula that will be our starting point for transformations to express the variation in terms of the data at infinity. 
Parameterization by the data at infinity. Let us now recall that, given the data $I$, II on the boundary of $N$ one can introduce the first and second fundamental forms "at infinity" via (30). Conversely, knowing the fundamental forms $I^{*}, I^{*}$ "at infinity" one can recover the fundamental forms on $\partial N$ via (32). Our aim is to rewrite the variation (44) of the $\mathrm{W}$-volume in terms of the variations of the forms $I^{*}, I^{*}$.

Lemma 6.1. The first-order variation of $W$ can be expressed as

$$
\delta W(N)=-\frac{1}{4} \int_{\partial N}\left\langle\delta I^{*}-\frac{H^{*}}{2} \delta I^{*}, I^{*}\right\rangle d a^{*} .
$$

Proof. Clearly the first-order variation of $W$ contains two kinds of terms, those related to the first-order variation of $I^{*}$ and those coming from the first-order variation of $B^{*}$, which we call $\delta B^{*}$ here. We define $X:=\left(I^{*}\right)^{-1} \delta I^{*}$, and consider separately the terms which are linear in $X$ and those which are linear in $\delta B^{*}$. To simplify notations we use the notation " $O(X)$ " (resp. " $O\left(\delta B^{*}\right)$ ") to describe any term linear in $X$ (resp. in $\delta B^{*}$ ). We will be using repeatedly a simple formula valid for any two $2 \times 2$ matrices $A$ and $B$ :

$$
\operatorname{det}(A) \operatorname{tr}\left(A^{-1} B\right)=\operatorname{tr}(A) \operatorname{tr}(B)-\operatorname{tr}(A B) .
$$

We consider first the terms linear in $\delta B^{*}$. We already know that

$$
2 I=I^{*}\left(\left(E+B^{*}\right) \cdot\left(E+B^{*}\right) \cdot\right),
$$

it follows that

$$
2 \delta I=2 I^{*}\left(\left(E+B^{*}\right) \cdot, \delta B^{*}\right)+O(X)
$$

so that

$$
\langle\delta I, I\rangle=2 \operatorname{tr}\left(\left(E+B^{*}\right)^{-1} \delta B^{*}\right)+O(X) .
$$

Similarly, we know that

$$
2 I I=I^{*}\left(\left(E+B^{*}\right) \cdot\left(E-B^{*}\right) \cdot\right),
$$

it follows that

$$
2 \delta I I=I^{*}\left(\delta B^{*} \cdot,\left(E-B^{*}\right) \cdot\right)-I^{*}\left(\left(E+B^{*}\right) \cdot, \delta B^{*} \cdot\right)+O(X)
$$

and therefore that

$$
\langle\delta I I, I\rangle=\operatorname{tr}\left(\left(E+B^{*}\right)^{-1}\left(E-B^{*}\right)\left(E+B^{*}\right)^{-1} \delta B^{*}\right)-\operatorname{tr}\left(\left(E+B^{*}\right)^{-1} \delta B^{*}\right)+O(X) .
$$

Moreover

$$
H=\operatorname{tr}\left(\left(E+B^{*}\right)^{-1}\left(E-B^{*}\right)\right),
$$

and putting all terms together shows that

$$
\begin{gathered}
\left\langle\delta I I-\frac{H}{2} \delta I, I\right\rangle=-\operatorname{tr}\left(\left(E+B^{*}\right)^{-1} \delta B^{*}\right)+ \\
+\operatorname{tr}\left(\left(E+B^{*}\right)^{-1}\left(E-B^{*}\right)\left(E+B^{*}\right)^{-1} \delta B^{*}\right)-\operatorname{tr}\left(\left(E+B^{*}\right)^{-1}\left(E-B^{*}\right)\right) \operatorname{tr}\left(\left(E+B^{*}\right)^{-1} \delta B^{*}\right)+O(X) .
\end{gathered}
$$

The last two terms can be treated as the right-hand side of (46), the equation becomes:

$$
\left\langle\delta I I-\frac{H}{2} \delta I, I\right\rangle=-\operatorname{tr}\left(\left(E+B^{*}\right)^{-1} \delta B^{*}\right)-\operatorname{det}\left(\left(E+B^{*}\right)^{-1}\left(E-B^{*}\right)\right) \operatorname{tr}\left(\left(E-B^{*}\right)^{-1} \delta B^{*}\right)+O(X) .
$$

We now apply (46) to each of the two terms on the right-hand side and get for the above quantity:

$$
\begin{array}{r}
\frac{\operatorname{tr}\left(\left(E+B^{*}\right) \delta B^{*}\right)-\operatorname{tr}\left(E+B^{*}\right) \operatorname{tr}\left(\delta B^{*}\right)-\operatorname{tr}\left(E-B^{*}\right) \operatorname{tr}\left(\delta B^{*}\right)+\operatorname{tr}\left(\left(E-B^{*}\right) \delta B^{*}\right)}{\operatorname{det}\left(E+B^{*}\right)}+O(X) \\
=\frac{2 \operatorname{tr}\left(\delta B^{*}\right)-4 \operatorname{tr}\left(\delta B^{*}\right)}{\operatorname{det}\left(E+B^{*}\right)}+O(X)=\frac{-2 \operatorname{tr}\left(\delta B^{*}\right)}{\operatorname{det}\left(E+B^{*}\right)}+O(X) .
\end{array}
$$


The part which is linear in $X$ can be computed in a similar way. First note that, by definition of $X$,

$$
\langle\delta I, I\rangle=\operatorname{tr}(X)+O\left(\delta B^{*}\right),
$$

while

$$
\delta I I=I^{*}\left(\left(E+B^{*}\right) \cdot, X\left(E-B^{*}\right) \cdot\right)+O\left(\delta B^{*}\right),
$$

so that

$$
\langle\delta I, I\rangle=\operatorname{tr}\left(\left(E+B^{*}\right)^{-1} X\left(E-B^{*}\right)\right)+O\left(\delta B^{*}\right) .
$$

It follows, using (46) and forgetting all terms linear in $\delta B^{*}$, that

$$
\begin{aligned}
\langle\delta I I-(H / 2) \delta I, I\rangle & =\operatorname{tr}\left(\left(E+B^{*}\right)^{-1} X\left(E-B^{*}\right)\right)-(1 / 2) \operatorname{tr}\left(\left(E+B^{*}\right)^{-1}\left(E-B^{*}\right)\right) \operatorname{tr}(X) \\
& =\frac{1}{\operatorname{det}\left(E+B^{*}\right)}\left(\operatorname{tr}\left(E+B^{*}\right) \operatorname{tr}\left(X\left(E-B^{*}\right)\right)-\operatorname{tr}\left(\left(E+B^{*}\right) X\left(E-B^{*}\right)\right)\right. \\
& \left.-(1 / 2) \operatorname{tr}\left(E+B^{*}\right) \operatorname{tr}\left(E-B^{*}\right) \operatorname{tr}(X)+(1 / 2) \operatorname{tr}\left(E-\left(B^{*}\right)^{2}\right) \operatorname{tr}(X)\right) \\
& =\frac{1}{\operatorname{det}\left(E+B^{*}\right)}\left(\operatorname{tr}\left(E+B^{*}\right) \operatorname{tr}\left(X\left(E-B^{*}\right)\right)-\operatorname{tr}\left(\left(E-\left(B^{*}\right)^{2}\right) X\right)\right. \\
& \left.-(1 / 2)\left(\operatorname{tr}(E)^{2}-\operatorname{tr}\left(B^{*}\right)^{2}\right) \operatorname{tr}(X)+(1 / 2) \operatorname{tr}\left(E-\left(B^{*}\right)^{2}\right) \operatorname{tr}(X)\right) .
\end{aligned}
$$

The terms involving $\left(B^{*}\right)^{2}$ can be replaced using the fact that $\left(B^{*}\right)^{2}-\operatorname{tr}\left(B^{*}\right) B^{*}+\operatorname{det}\left(B^{*}\right) E=0$, so that $\operatorname{tr}\left(\left(B^{*}\right)^{2}\right)=\operatorname{tr}\left(B^{*}\right)^{2}-2 \operatorname{det}\left(B^{*}\right)$. It follows that, still forgetting all terms which are linear in $\delta B^{*}$, we have

$$
\begin{aligned}
\langle\delta I-(H / 2) \delta I, I\rangle & =\frac{1}{\operatorname{det}\left(E+B^{*}\right)}\left(\operatorname{tr}\left(E+B^{*}\right) \operatorname{tr}\left(X\left(E-B^{*}\right)\right)-\operatorname{tr}\left(\left(1+\operatorname{det}\left(B^{*}\right)\right) X-\operatorname{tr}\left(B^{*}\right) B^{*} X\right)\right. \\
& \left.-(1 / 2)\left(4-\operatorname{tr}\left(B^{*}\right)^{2}\right) \operatorname{tr}(X)+(1 / 2)\left(2-\operatorname{tr}\left(B^{*}\right)^{2}+2 \operatorname{det}\left(B^{*}\right)\right) \operatorname{tr}(X)\right) \\
& =\frac{1}{\operatorname{det}\left(E+B^{*}\right)}\left(\left(2+\operatorname{tr}\left(B^{*}\right)\right)\left(\operatorname{tr}(X)-\operatorname{tr}\left(B^{*} X\right)\right)-\left(1+\operatorname{det}\left(B^{*}\right)\right) \operatorname{tr}(X)+\operatorname{tr}\left(B^{*}\right) \operatorname{tr}\left(B^{*} X\right)\right. \\
& \left.+\left(\operatorname{det}\left(B^{*}\right)-1\right) \operatorname{tr}(X)\right) \\
& =\frac{\operatorname{tr}\left(B^{*}\right) \operatorname{tr}(X)-2 \operatorname{tr}\left(B^{*} X\right)}{\operatorname{det}\left(E+B^{*}\right)} .
\end{aligned}
$$

Putting together the terms in $X$ and the terms in $\delta B^{*}$, and using the fact that $d a^{*}=(1 / 2) \operatorname{det}\left(E+B^{*}\right) d a$ by definition of $I^{*}$, we find that:

$$
\langle\delta I I-(H / 2) \delta I, I\rangle=-\left(\operatorname{tr}\left(\delta B^{*}\right)+\operatorname{tr}\left(B^{*} X\right)-(1 / 2) \operatorname{tr}\left(B^{*}\right) \operatorname{tr}(X)\right)\left(d a^{*} / d a\right)=-\left\langle\delta I^{*}-\left(H^{*} / 2\right) \delta I^{*}, I^{*}\right\rangle\left(d a^{*} / d a\right),
$$

and the result clearly follows.

Formula (45) looks very much like the original formula (44), except for the minus sign and the fact that the quantities at infinity are used. The fact that we have got the same variational formula as in terms of the data on $\partial N$ is not too surprising. Indeed, the variational formula (45) was obtained from (44) by applying the transformation (32). As it is clear from (30), this transformation applied twice gives the identity map. In view of this, it is hard to think of any other possibility for the variational formula in terms of $\delta I^{*}, \delta I^{*}$ except being given by the same expression (44), apart from maybe with a different sign. This is exactly what we see in (45).

There is another expression of the first-order variation of $W$, dual to (41), which will be useful below.

Corollary 6.2. The first-order variation of $W$ can also be expressed as

$$
\delta W=-\frac{1}{4} \int_{\partial N} \delta H^{*}+\left\langle\delta I^{*}, I_{0}^{*}\right\rangle d a^{*},
$$

where $I_{0}^{*}$ is the traceless part (for $I^{*}$ ) of $I^{*}$. 


\section{Conformal variations of the metric at infinity}

In this section we use Corollary 6.2 to prove one simple corollary. Thus, we show that, when varying the $\mathrm{W}$-volume with the area of the boundary defined by the $I^{*}$ metric kept fixed, the variational principle implies the metric $I^{*}$ to have constant negative curvature. The variations we consider in this section do not change the conformal structure of the metric $I^{*}$, and thus do not change the manifold $M$. Geometrically they correspond to small movements of the surface $\partial N$ inside the fixed manifold $M$.

First variation. We consider in this section a conformal deformation of the metric $I^{*}$, i.e., $\delta I^{*}=2 u I^{*}$, where $u$ is some function on $\partial N$. Clearly for such variations $\left\langle\delta I^{*}, I_{0}^{*}\right\rangle=0$, precisely because $I_{0}^{*}$ is traceless.

Let us consider the following functional

$$
F(N)=W(N)-\frac{\lambda}{4} \int_{\partial N} d a^{*}
$$

appropriate for finding an extremum of the $\mathrm{W}$-volume with the area computed using the metric $I^{*}$ kept fixed. The first variation of this functional gives, using Corollary 6.2

$$
\delta F=-\frac{1}{4} \int_{\partial N}\left(\delta H^{*}\right) d a^{*}-\frac{\lambda}{4} \int_{\partial N} 4 u d a^{*}=\frac{1}{4} \int_{\partial N}\left(\delta K^{*}\right) d a^{*}-\frac{\lambda}{4} \int_{\partial N} 4 u d a^{*} .
$$

But

$$
\delta \int_{\partial N} K^{*} d a^{*}=\int_{\partial N}\left(\delta K^{*}\right)+4 u K^{*} d a^{*}=0
$$

by the Gauss-Bonnet formula, so that

$$
\delta F=\int_{\partial N}\left(-u K^{*}-u \lambda\right) d a^{*} .
$$

It follows that critical points of $F$ are characterized by the fact that $K^{*}=-\lambda$.

Second variation. In this paragraph we would like to verify whether the extremum found above is maximum or minimum. It is easy to compute the formula for the second variation, but for the sake of simplicity we only do it here for conformal deformations of $I^{*}$, corresponding to movements of $\partial N$.

According to the formula found right above, the gradient of $F$ on the space of metrics conformal to $I^{*}$ is simply $D F=-\left(K^{*}+\lambda\right) / 4$. However a well-known formula on the conformal deformations of metrics (see e.g. chapter 1 of [Bes87]) indicates that, under a conformal deformation $\delta I^{*}=2 v I^{*}$,

$$
\delta K^{*}=-2 v K^{*}+\Delta v,
$$

where $\Delta$ is the Laplacian for $I^{*}$. It follows directly that the Hessian of $F$ at a critical point is given by

$$
(\operatorname{Hess}(F))\left(2 u I^{*}, 2 v I^{*}\right)=\int_{\partial N} 2 K^{*} u v-(\Delta v) u d a^{*}=\int_{\partial N}-2 \lambda u v-\langle d u, d v\rangle d a^{*} .
$$

This quantity is negative definite (because $\lambda=-K^{*}>0$ by Gauss-Bonnet) so that the critical points of $F$ are local maxima.

\section{The renormalized volume as a function on Teichmüller space}

In this section we consider the renormalized volume as a function over the Teichmüller space of $\partial N$; in other terms, for each conformal class on $\partial N$, we consider the extremum of $W$ over metrics of given area within this conformal class. We have seen in the previous section that this extremum is obtained at the (unique) constant curvature metric. The main goal here is to recover by simple differential geometric methods important results of McMullen McM00 - concerning his "quasi-Fuchsian reciprocity" - and Takhtajan and Zograf TZ87, Takhtajan and Teo [TT03] - showing that the renormalized volume provides a Kähler potential for the WeilPetersson metric. So the "volume" that we consider here is now defined as follows. 
Definition 8.1. Let $g$ be a convex co-compact hyperbolic metric on $M$, and let $c \in \mathcal{T}_{\partial M}$ be the conformal structure induced on $\partial_{\infty} M$. We call $W_{M}(c)$ the value of $W$ on the equidistant foliation of $M$ near infinity for which $I^{*}$ has constant curvature -1 .

In other terms, by the results obtained in the previous section, $W_{M}(c)$ is the maximum of $W$ over the metrics at infinity which have the same area as a hyperbolic metric, for each boundary component of $M$. Throughout this section the metric at infinity $I^{*}$ that we consider is the hyperbolic metric, while the second fundamental form at infinity, $I^{*}$, is uniquely determined by the choice that $I^{*}$ is hyperbolic. Its traceless part is denoted by $I_{0}^{*}$.

The second fundamental form at infinity as the real part of a HQD. It is interesting to remark that, in the context considered here - when $I^{*}$ has constant curvature - the second fundamental form at infinity has a complex interpretation. This can be compared with the same phenomenon, discovered by Hopf [Hop51, for the second fundamental form of constant mean curvature surfaces in 3-dimensional constant curvature spaces.

Lemma 8.2. When $K^{*}$ is constant, $I_{0}^{*}$ is the real part of a quadratic holomorphic differential (for the complex structure associated to $I^{*}$ ) on $\partial_{\infty} M$. This holomorphic quadratic differential is given explicitly by (38).

Proof. By definition $I_{0}^{*}$ is traceless, which means that it is at each point the real part of a quadratic differential: $I_{0}^{*}=\operatorname{Re}(h)$. Moreover, we have seen in Remark [5.5 that $B^{*}$ satisfies the Codazzi equation, $d^{\nabla^{*}} B^{*}=0$. It follows as for constant mean curvature surfaces (see e.g. [KS05]) that $h$ is holomorphic relative to the complex structure of $I^{*}$.

The second fundamental form as a Schwarzian derivative. The next step is that, for each boundary component $\partial_{i} M$ of $M, I_{0 i}^{*}$ is actually the real part of the Schwarzian derivative of a natural equivariant map between the hyperbolic plane (with its canonical complex projective structure) to $\partial_{i} M$ with its complex projective structure induced by the hyperbolic metric on $M$. In the terminology used by McMullen McM00, $I_{0 i}^{*}$ is the difference between the complex projective structure at infinity on $\partial_{i} M$ and the Fuchsian projective structure on $\partial_{i} M$.

A simple way to prove this assertion is to use the formula (38) for the holomorphic quadratic differential $\theta$ whose real part gives the traceless part of $I^{*}$. The Liouville field $\phi$ that enters into this formula can be simply expressed in terms of the conformal map from $\partial_{i} M$ to the hyperbolic plane. It is then a standard and simple computation to verify that $\theta$ is equal to the Schwarzian derivative of this map, see e.g. [TZ87. To make this paper self-contained we decided to include yet a different, more geometric proof, which is spelled out in the appendix. The proof we give is elementary, based on the conformal factor between the $I^{*}$ metrics on corresponding surfaces in two foliations. It can be compared to the argument used in [McM00].

To state the result, let us call $\sigma_{F}$ the "Fuchsian" complex projective structure on $\partial_{i} M$, obtained by applying the Poincaré uniformization theorem to the conformal metric at infinity on $\partial_{i} M$. The universal cover of $\partial_{i} M$, with the complex projective structure lifted from $\sigma_{F}$, is projectively equivalent to a disk in $\mathbb{C} P^{1}$. We also call $\sigma_{Q F}$ the projective structure induced on $\partial_{i} M$ by the hyperbolic metric on $M$. Here " $Q F$ " stands for quasiFuchsian (while $M$ is only supposed to be convex co-compact), this notation is used to keep close to the notation in [McM00. The map $\phi:\left(\partial_{i} M, \sigma_{F}\right) \rightarrow\left(\partial_{i} M, \sigma_{Q F}\right)$ which is isometric for the hyperbolic metrics on $\partial_{i} M$, is conformal but not projective between $\left(\partial_{i} M, \sigma_{F}\right)$ and $\left(\partial_{i} M, \sigma_{Q F}\right)$, so we can consider its Schwarzian derivative $\mathcal{S}(\phi)$.

Lemma 8.3. $I_{0}^{*}=-\operatorname{Re}(\mathcal{S}(\phi))$.

It is possible to reformulate this statement slightly by setting $\theta_{i}:=\mathcal{S}(\phi)$ (this is analogous to the notations used in [McM00, the index $i$ is useful to recall that this quantity is related to $\left.\partial_{i} M\right)$. Then $\theta_{i}$ is a quadratic holomorphic differential (QHD) on $\partial_{i} M$, and, still using the notations in McM00, the definition of $\theta_{i}$ can be rephrased as: $\theta_{i}=\sigma_{Q F}-\sigma_{F}$. The Lemma can then be written as: $I_{0 i}^{*}=\operatorname{Re}\left(\theta_{i}\right)$. A geometric proof of this lemma is given in the appendix. 
Remark 8.4. Note that $\theta_{i}$ can also be considered as a complex-valued 1-form on the Teichmüller space of $\partial_{i} M$. Indeed, it is well known that the cotangent vectors to $\mathcal{T}_{S}$, where $S$ is a Riemann surface, can be described as holomorphic quadratic differentials $q$ on $S$. The pairing with a tangent vector (Beltrami differential $\mu$ ) is given by the integral of $q \mu$ over $S$. The complex structure on $\mathcal{T}_{S}$ can then be described as follows: the image of the cotangent vector $q$ under the action of the complex structure $J$ is simply $J(q)=i q$. Another, more geometric way to state the action of $J$ is to note that it exchanges the horizontal and vertical foliations of $q$. Thus, holomorphic quadratic differentials $q$ on $S$ are actually holomorphic 1-forms on $\mathcal{T}_{S}$.

The second fundamental form as the differential of $W_{M}$. There is another simple interpretation of the traceless part of the second fundamental form at infinity.

Lemma 8.5. The differential $d W_{M}$ of the renormalized volume $W_{M}$, as a 1-form over the Teichmüller space of $\partial M$, is equal to $(-1 / 4) I_{0}^{*}$.

Proof. This is another direct consequence of Corollary 6.2 because, as one varies $I^{*}$ among hyperbolic metrics, $H^{*}$ (which is equal to $K^{*}$ ) remains equal to -1 , so that $\delta H^{*}=0$.

Corollary 8.6. $\theta_{i}=-4 \partial W_{M}$.

Proof. This follows directly from the lemma, since we already know that $\theta_{i}$ is a holomorphic differential.

Remark 8.7. We would like to emphasize how much simpler is the proof given above than that given in TZ87, TT03. Unlike in these references, which obtain the above result on the gradient of $W_{M}$ using an involved computation, the Corollary 6.2 implies this result in one line. This demonstrates the strength of the geometric method used here.

McMullen's quasi-Fuchsian reciprocity. We can now recover McMullen's quasi-Fuchsian reciprocity as a simple consequence of the proved above relation between $W_{M}$ and the second fundamental form at infinity. In the context considered here, it is just a consequence of the fact that the Hessian of a function is symmetric.

From this point and until the end of this section we suppose that $M$ is quasi-Fuchsian. It has two boundary components, which we call $\partial_{-} M$ and $\partial_{+} M$, which are homeomorphic. The renormalized volume $W_{M}$ is now a function on the space of quasi-Fuchsian metrics on $M$, which by the Bers theorem is $\mathcal{T}_{\partial_{-} M} \times \mathcal{T}_{\partial_{+} M}$.

Let $\left(c_{-}, c_{+}\right) \in \mathcal{T}_{\partial_{-} M} \times \mathcal{T}_{\partial_{+} M}$, they define a unique hyperbolic metric on $M$, and therefore a complex projective structure $\sigma_{Q F,+}\left(c_{-}, c_{+}\right)$(resp. $\left.\sigma_{Q F,-}\left(c_{-}, c_{+}\right)\right)$on $\partial_{+} M$ (resp. $\left.\partial_{-} M\right)$. Using the Schwarzian derivative construction this can be used to define a QHD $\beta_{+}\left(c_{-}, c_{+}\right)$(resp. $\left.\beta_{+}\left(c_{-}, c_{+}\right)\right)$on $\partial_{+} M\left(\right.$ resp. $\left.\partial_{-} M\right)$ as

$$
\beta_{+}\left(c_{-}, c_{+}\right)=\sigma_{Q F,+}\left(c_{-}, c_{+}\right)-\sigma_{F}\left(c_{+}\right)
$$

and respectively

$$
\beta_{-}\left(c_{-}, c_{+}\right)=\sigma_{Q F,-}\left(c_{-}, c_{+}\right)-\sigma_{F}\left(c_{-}\right) .
$$

Then $\beta_{+}\left(c_{-}, c_{+}\right)$is a HQD on $\partial_{+} M$, and can therefore be identified with an element of $T_{c_{+}}^{*} \mathcal{T}_{\partial_{+} M}$. So $c_{-}$ determines a cotangent vector field $\beta_{+}\left(c_{-}, \cdot\right)$ on $\mathcal{T}_{\partial_{+} M}$, and similarly $c_{+}$determines a cotangent vector field $\beta_{-}\left(\cdot, c_{+}\right)$on $\mathcal{T}_{\partial_{+} M}$. By Lemma 8.3 above,

$$
I_{0,+}^{*}=-\operatorname{Re}\left(\beta_{+}\left(c_{-}, c_{+}\right)\right), \Pi_{0,-}^{*}=-\operatorname{Re}\left(\beta_{-}\left(c_{-}, c_{+}\right)\right) .
$$

Yet another way to state this relationship is that

$$
\forall v_{+} \in T_{c_{+}} \mathcal{T}_{\partial_{+} M}, \beta_{+}\left(c_{-}, c_{+}\right)\left(v_{+}\right)=-\left\langle I_{0,+}^{*}, v_{+}\right\rangle
$$

and similarly for $\beta_{-}$. Using Lemma 8.5] $\beta_{+}$and $\beta_{-}$can be expressed in terms of $W_{M}$ as follows:

$$
\forall v_{+} \in T_{c_{+}} \mathcal{T}_{\partial_{+} M}, \beta_{+}\left(c_{-}, c_{+}\right)\left(v_{+}\right)=4 d W_{M}\left(\left(0, v_{+}\right)\right),
$$


and similarly for $\beta_{-}$. Here $W_{M}$ is considered as a function on $\mathcal{T}_{\partial_{-} M} \times \mathcal{T}_{\partial_{+} M}$, and $\left(0, v_{+}\right)$is a vector tangent to $\mathcal{T}_{\partial_{-} M} \times \mathcal{T}_{\partial_{+} M}$. $\partial_{-} M$

We can now consider the differential of the $\beta_{+}\left(\cdot, c_{+}\right)$considered as a function of the conformal structure on

$$
D \beta_{+}\left(\cdot, c_{+}\right): T_{c_{-}} \mathcal{T}_{\partial_{-} M} \rightarrow T_{c_{+}}^{*} \mathcal{T}_{\partial_{+} M}
$$

and of $\beta_{-}\left(c_{-}, \cdot\right)$, considered as a function of the conformal structure on $\partial_{+} M$

$$
D \beta_{-}\left(c_{-}, \cdot\right): T_{c_{+}} \mathcal{T}_{\partial_{+} M} \rightarrow T_{c_{-}}^{*} \mathcal{T}_{\partial_{-} M} .
$$

Theorem 8.8 (McMullen's quasi-Fuchsian reciprocity McM00]). The maps $D \beta_{+}\left(\cdot, c_{+}\right)$and $D \beta_{-}\left(c_{-}, \cdot\right)$ are adjoint to each other.

Proof. Let $v_{-} \in T_{c_{-}} \mathcal{T}_{\partial_{-} M}$ and $v_{+} \in T_{c_{+}} \mathcal{T}_{\partial_{+} M}$. Then

$$
\left\langle D \beta_{+}\left(c_{-}, c_{+}\right)\left(v_{-}, 0\right), v_{+}\right\rangle=4\left(D_{\left(v_{-}, 0\right)} d W_{M}\right)\left(\left(0, v_{+}\right)\right)=4\left(\operatorname{Hess}\left(W_{M}\right)\right)\left(\left(v_{-}, 0\right),\left(0, v_{+}\right)\right) .
$$

(Note that the Hessian here can be considered without reference to a specific connection, because $\left(v_{-}, 0\right)$ and $\left(0, v_{+}\right)$are tangent to $\mathcal{T}_{\partial_{-} M}$ and $\mathcal{T}_{\partial_{+} M}$ respectively.) The symmetry in the right-hand side shows quite clearly that

$$
\left\langle D \beta_{+}\left(c_{-}, c_{+}\right)\left(v_{-}, 0\right), v_{+}\right\rangle=\left\langle D \beta_{-}\left(c_{-}, c_{+}\right)\left(0, v_{+}\right), v_{-}\right\rangle,
$$

which is precisely the statement of the theorem.

The renormalized volume as a Kähler potential. Finally we show here how to recover in this manner the result of Takhtajan and Teo [TT03] stating that the renormalized volume $W_{M}$ with $c_{-}$fixed is a Kähler potential for the Weil-Petersson metric on $\mathcal{T}_{\partial_{+} M}$. To simplify notations a little, we set $\theta_{c_{-}}:=\beta_{+}\left(c_{-}, \cdot\right)$. Since we already know that $\theta_{c_{-}}=-4 \partial W_{M}$, we only need to prove that $\bar{\partial}\left(i \theta_{c_{-}}\right)=-2 \omega_{W P}$, where $\omega_{W P}$ is the Kähler form of the Weil-Petersson metric on $\mathcal{T}_{\partial_{+} M}$.

An important part of the argument is that $d \theta_{c_{-}}$, as a 2-form on $\mathcal{T}_{\partial_{+} M}$, does not depend on $c_{-}$. This appears as Theorem 7.2 in McMullen's paper [McM00. We include a proof for completeness, following the proof given in McM00.

Proposition 8.9. The differential $d \theta_{c_{-}}$, considered as a complex-valued 2-form on $\mathcal{T}_{\partial_{+} M}$, does not depend on $c_{-}$.

Proof. Let $v_{-} \in T_{c_{-}} \mathcal{T}_{\partial_{-} M}$, we want to show that the corresponding first-order variation $D_{v_{-}}\left(d \theta_{c_{-}}\right)$of $d \theta_{c_{-}}$ vanishes. This will follow from the fact that the first-order variation of $\theta_{c_{-}}$corresponding to $v_{-}, D_{v_{-}} \theta_{c_{-}}$, is the differential of a function defined on $\mathcal{T}_{\partial_{+} M}$, namely the function $f_{v_{-}}$defined by

$$
f_{v_{-}}\left(c_{+}\right)=\left\langle\beta_{-}\left(c_{-}, c_{+}\right), v_{-}\right\rangle,
$$

where $\langle$,$\rangle is the WP pairing.$

The fact that $D_{v_{-}} \theta_{c_{-}}=d f_{v_{-}}$can be proved by evaluating both sides on a vector $v_{+} \in T_{c_{+}} \mathcal{T}_{\partial_{+} M}$ and using the quasi-Fuchsian reciprocity.

$$
\left\langle D_{v_{-}} \theta_{c_{-}}, v_{+}\right\rangle=\left\langle D \beta_{+}\left(c_{-}, c_{+}\right)\left(v_{-}, 0\right), v_{+},\right\rangle=\left\langle D \beta_{-}\left(c_{-}, c_{+}\right)\left(0, v_{+}\right), v_{-}\right\rangle=d f_{v_{-}}\left(v_{+}\right) .
$$

It clearly follows that $d \theta_{c_{-}}$, as a 2 -form on $\mathcal{T}_{\partial_{+} M}$, does not depend on $c_{-}$.

That $W_{M}$ is a Kähler potential is then reduced to a simple computation in the Fuchsian situation.

Proposition 8.10. Suppose that $M$ is a Fuchsian manifold, with $c_{+}=c_{-}$. Let $I^{*}$ be the hyperbolic metric in the conformal class $c_{+}$. Under a first-order deformation which does not change $c_{-}$, the variation of $I^{*}$ and $I_{0}^{*}$ on $\partial_{+} M$ are related by:

$$
\delta I_{0}^{*}=-\delta I^{*} .
$$


Proof. It follows from the constructions in the appendix that $B^{*}=(1 / 2) E$. Under a first-order variation we have that

$$
\begin{gathered}
\delta I^{*}=(1 / 2) \delta I^{*}+I^{*}\left(\delta B^{*} \cdot, \cdot\right), \\
\delta I I^{*}=\delta I^{*}\left(B^{*} \cdot, B^{*} \cdot\right)+2 I^{*}\left(\delta B^{*} \cdot, B^{*} \cdot\right)=(1 / 4) \delta I^{*}+I^{*}\left(\delta B^{*} \cdot, \cdot\right),
\end{gathered}
$$

so that $\delta I^{*}=2 \delta^{*} I^{*}-\delta I^{*}$.

But III* $^{*}$ is the hyperbolic metric in the conformal class $c_{-}$and therefore it does not change under the deformation, so that $2 \delta I^{*}=\delta I^{*}$.

Moreover, since $I^{*}$ remains hyperbolic, $H^{*}=\operatorname{tr}_{I^{*}} I^{*}=1$, so that $I_{0}^{*}=I^{*}-(1 / 2) I^{*}$, and so

$$
\delta \Pi_{0}^{*}=\delta I^{*}-(1 / 2) \delta I^{*}=-\delta I^{*} .
$$

We can reformulate this statement by calling $\theta_{R}:=\operatorname{Re}\left(\theta_{c_{-}}\right)$, so that, by Lemma $8.3, \theta_{R}(X)=-\left\langle X, I_{0}^{*}\right\rangle$. Using the previous proposition, this can the be stated as

$$
\left(D_{X} \theta_{R}\right)(Y)=\langle X, Y\rangle_{W P},
$$

where $D$ is the Levi-Cività connection of the Weil-Petersson metric on $\mathcal{T}_{\partial_{+} M}$.

We can now compute explicitly an expression of $\bar{\partial} \theta_{c_{-}}$, denoting by $J$ the complex structure on $\mathcal{T}_{\partial_{-}} M$.

$$
\begin{aligned}
\bar{\partial} \theta_{c_{-}}(X, Y) & =\left(D_{X} \theta_{c_{-}}\right)(Y)+i\left(D_{J X} \theta_{c_{-}}\right)(Y) \\
& =\left(D_{X} \theta_{R}\right)(Y)-i\left(D_{X} \theta_{R}\right)(J Y)+i\left(\left(D_{J X} \theta_{R}\right)(Y)-i\left(D_{J X} \theta_{R}\right)(J Y)\right) \\
& =\langle X, Y\rangle-i\langle X, J Y\rangle+i\langle J X, Y\rangle+\langle J X, J Y\rangle \\
& =2(\langle X, Y\rangle-i\langle X, J Y\rangle) .
\end{aligned}
$$

This means precisely that $\bar{\partial} \theta_{c_{-}}(X, J X)=2 i\|X\|_{W P}^{2}$, and we recover the result of Takhtajan and Teo TT03. that $W_{M}$ is a Kähler potential for the Weil-Petersson metric.

\section{A Appendix: A geometric proof of $I_{0}^{*}=-\operatorname{Re}(\mathcal{S}(\phi))$.}

The proof is based on some preliminary but simple arguments on the conformal factor relating corresponding surfaces in the equidistant foliations of a quasi-Fuchsian and a Fuchsian manifold.

We consider a point $x_{0} \in \partial_{i} M$. For each point $x$ in some neighborhood of $x_{0}$ there is then a unique geodesic $\gamma_{x}$, orthogonal to the leaves of the foliation by the surfaces $\Sigma_{\rho}$ already introduced above, with endpoint $x$. In addition to $M$, we also consider the "Fuchsian" manifold $M^{\prime}$ with boundary at infinity equal to the disjoint union of two copies of $\partial_{i} M$ (with its conformal metric). So $M^{\prime}$ is topologically $\partial_{i} M \times \mathbb{R}$, with the hyperbolic metric

$$
d \rho^{2}+\cosh ^{2}(\rho) I^{*},
$$

where $I^{*}$ is the hyperbolic metric on $\partial_{i} M$. Using this construction we can identify $\partial_{i} M$ also with the "upper" boundary component of $M^{\prime}$. To avoid ambiguities we call this upper boundary component $\partial_{i} M^{\prime}$, and consider the map $\phi$ defined above as a map between $\partial_{i} M^{\prime}$ and $\partial_{i} M$, and call $x_{0}^{\prime}$ the point corresponding to $x_{0}$ on $\partial_{i} M^{\prime}$. There is a canonical foliation on $M^{\prime}$, given by the level surfaces $\Sigma_{\rho}^{\prime}$ of $\rho$ in (54), to which we can associate a metric $I^{\prime *}$ and a second fundamental form $I^{\prime *}$ at infinity on $\partial_{i} M^{\prime}$. Taking the right choice of foliation near each boundary component (which involves an affine transformation on $\rho$ ) leads to $2 I^{\prime *}=I^{\prime *}=2 \phi^{*} I^{*}$, so that $B^{\prime *}=(1 / 2) E$.

We now consider the geodesics $\gamma_{x_{0}}$ (in $M$ ) and $\gamma_{x_{0}^{\prime}}^{\prime}$ (in $M^{\prime}$ ), with their respective parameterizations. There is a unique hyperbolic isometry $\Phi_{0}$ sending $\gamma_{x_{0}^{\prime}}^{\prime}$ to $\gamma_{x_{0}}$, preserving the parameterization by $\rho$, and such that the differential at $x_{0}^{\prime}$ of the boundary map $\phi_{0}: \partial_{\infty} H^{3} \rightarrow \partial_{\infty} H^{3}$ is tangent to the isometry between $I^{\prime *}$ and $I^{*}$. 
Then $\phi_{0}$ is a complex projective map but it is not an isometry between $I^{*}$ and $I^{*}$ (although its differential at $x_{0}^{\prime}$ is isometric).

We will consider the map $\phi_{0}^{-1} \circ \phi: \partial_{i} M^{\prime} \rightarrow \partial_{i} M^{\prime}$, and show that it is tangent to the identity at order 2 at $x_{0}^{\prime}$; this shows that $\phi_{0}$ is the "best" approximation of $\phi$ at $x_{0}^{\prime}$ by a projective map, and therefore that the third derivative of $\phi_{0}^{-1} \circ \phi$ at $x_{0}^{\prime}$ determines the Schwarzian derivative $\mathcal{S}(\phi)$ at $x_{0}^{\prime}$ (see e.g. [OS98, (1.3)). By construction,

$$
\left(\phi_{0}^{-1} \circ \phi\right)_{*} I^{*}=\left(\phi_{0}^{-1}\right)_{*}\left(\phi_{*} I^{\prime *}\right)=\left(\phi_{0}^{-1}\right)_{*} I^{*}=\phi_{0}^{*} I^{*}
$$

so $\phi_{0}^{-1} \circ \phi$ is an isometry between $I^{* *}$ and $\phi_{0}^{*} I^{*}$. Those two metrics on $\partial_{i} M^{\prime}$ are conformal, we will show that the Hessian at $x_{0}^{\prime}$ of the conformal factor is $\phi^{*} I_{0}^{*}$, and deduce from this the proof of Lemma 8.3 ,

Proposition A.1. The shape operator of $\Sigma_{\rho}$ is given by $B_{\rho}=\left(E+e^{-2 \rho} B^{*}\right)^{-1}\left(E-e^{-2 \rho} B^{*}\right)$, so that, as $\rho \rightarrow \infty$, $B_{\rho}-E \simeq-2 e^{-2 \rho} B^{*}$.

Note that $B_{\rho}$ and $B^{*}$ are defined at different points $\left(B_{\rho}\right.$ on $\Sigma_{\rho}$ and $B^{*}$ on $\left.\partial_{i} M\right)$ which are implicitly identified here through the Gauss flow.

Proof. We have already computed above that

$$
I_{\rho}=(1 / 2)\left(e^{2 \rho} I^{*}+2 I^{*}+e^{-2 \rho} I^{*}\right)=(1 / 2) I^{*}\left(\left(e^{\rho} E+e^{-\rho} B^{*}\right) \cdot\left(e^{\rho} E+e^{-\rho} B^{*}\right) \cdot\right) .
$$

It follows that

$$
I_{\rho}=\frac{1}{2} \frac{d I_{\rho}}{d \rho}=\frac{1}{2}\left(e^{2 \rho} I^{*}-e^{-2 \rho} \Pi^{*}\right)=\frac{1}{2} I^{*}\left(\left(e^{\rho} E+e^{-\rho} B^{*}\right) \cdot,\left(e^{\rho} E-e^{-\rho} B^{*}\right) \cdot\right),
$$

and the result is a direct consequence.

Definition A.2. For each $x$ in some neighborhood of $x_{0}$ and all $\rho$ large enough, we call $d_{\rho}(x)$ the oriented distance, along $\gamma_{x}$, between its intersection with $\Phi_{0}\left(\Sigma_{\rho}^{\prime}\right)$ and with $\Sigma_{\rho}$. Then we define $d_{\infty}(x):=\lim _{\rho \rightarrow \infty} d_{\rho}(x)$.

Proposition A.3. The limit distance $d_{\infty}$ vanishes at $x_{0}$ along with its derivative, and its Hessian at $x_{0}$ is equal to $\mathrm{II}_{0}^{*}$.

Proof. By construction, $\gamma_{x}$ is orthogonal to $\Sigma_{\rho}$. But $\Phi_{0}\left(\Sigma_{\rho}^{\prime}\right)$ is tangent to $\Sigma_{\rho}$ at their common intersection with $\gamma_{x_{0}}$, so that $d_{\rho}$ vanishes along with its derivative at $x_{0}$.

Moreover, the second-order variation of $d_{\rho}$ at $x_{0}$ is determined by the difference between the shape operators at their intersection point with $\gamma_{x}$ (for $x$ close to $x_{0}$ ) of $\Phi_{0}\left(\Sigma_{\rho}^{\prime}\right)$ and of $\Sigma_{\rho}$. But, by Proposition A.1 applied to $\Sigma_{\rho}$ and to $\Sigma_{\rho}^{\prime}$, the dominant term in the difference between those shape operators is

$$
B_{\rho}^{\prime}-B_{\rho} \simeq e^{-2 \rho}\left(2 B^{*}-E\right) .
$$

Integrating this second derivative of $d_{\rho}$ shows that the Hessian of $d_{\rho}$ is equal to $I_{\rho}\left(e^{-2 \rho}\left(2 B^{*}-E\right) \cdot, \cdot\right)$, which is equivalent to $(1 / 2)\left(2 I^{*}-I^{*}\right)$, i.e., to $I_{0}^{*}$. The result then follows by taking the limit as $\rho \rightarrow \infty$.

Since $\phi_{0}$ is a projective map, it is conformal, and therefore the metrics $I^{*}$ and $\phi_{0}^{*} I^{*}$ on $\partial_{i} M^{\prime}$ are conformal, which means that there exists a function $u: \partial_{i} M^{\prime} \rightarrow \mathbb{R}$ such that $I^{\prime *}=e^{2 u} \phi_{0}^{*} I^{*}$.

Proposition A.4. $u\left(x_{0}^{\prime}\right)=0$ and $d u=0$ at $x_{0}^{\prime}$, while the Hessian of $u$ at $x_{0}^{\prime}$ is equal to $I_{0}^{*}$.

Proof. First note that by definition of $I^{*}$ it can be described in terms of the Gauss map, which we call $G_{\rho}$, from $\Sigma_{\rho}$ to $\partial_{i} M$, sending a point $x \in \Sigma_{\rho}$ to the endpoint at infinity of the geodesic ray starting from $x$ orthogonal to $\Sigma_{\rho}$ :

$$
I^{*}=(1 / 2) e^{-2 \rho} G_{\rho *}\left(I_{\rho}+2 I_{\rho}+I_{\rho}\right) \simeq 2 e^{-2 \rho} G_{*} I_{\rho} .
$$

The same can be said of $\Phi_{0}\left(\Sigma_{\rho}\right)$, using the Gauss map $G_{\rho}^{\prime}$ of $\Sigma_{\rho}^{\prime}$ :

$$
I^{\prime *} \simeq(1 / 2) e^{-2 \rho} G_{\rho *}^{\prime}\left(I_{\rho}^{\prime}+2 I_{\rho}^{\prime}+I I I_{\rho}^{\prime}\right) \simeq 2 e^{-2 \rho} G_{\rho *}^{\prime} I_{\rho}^{\prime} .
$$


Considering the image of $\Sigma_{\rho}^{\prime}$ by $\Phi_{0}$ and the behavior of the geodesic rays orthogonal to $\Phi_{0}\left(\Sigma_{\rho}^{\prime}\right)$ then shows that, in the neighborhood of $x_{0}$,

$$
\phi_{0 *}\left(G_{\rho *}^{\prime} I_{\rho}^{\prime}\right) \simeq e^{2 d_{\rho}} G_{\rho *} I_{\rho},
$$

and the result follows by taking the limit as $\rho \rightarrow \infty$.

Proof of Lemma 8.3. Let $f:=\phi_{0}^{-1} \circ \phi$. By construction the map $f: \partial_{i} M^{\prime} \rightarrow \partial_{i} M^{\prime}$ sends $x_{0}^{\prime}$ to $x_{0}^{\prime}$, and it is tangent to the identity at $x_{0}^{\prime}$. We have seen above that $f$ is an isometry between $I^{\prime *}$ and $\phi_{0}^{*} I^{*}$, so it follows from Proposition A.4 that $\left|f^{\prime}(z)\right|^{2}=e^{-2 u}$, where $u\left(x_{0}^{\prime}\right)=0, d u=0$ at $x_{0}^{\prime}$, and the Hessian of $u$ at $x_{0}^{\prime}$ is $\Pi_{0}^{*}$.

Since $\left(\partial_{i} M^{\prime}, I^{\prime *}\right)$ is hyperbolic, we can identify a neighborhood of $x_{0}^{\prime}$ with a neighborhood of 0 in the unit disk. Since $f$ is conformal it is then holomorphic, and can be written as:

$$
f(z)=z+\frac{b}{2} z^{2}+\frac{c}{6} z^{3}+o\left(z^{3}\right)
$$

with $b=f^{\prime \prime}(0)$ and $c=f^{\prime \prime \prime}(0)$, and clearly $\left|f^{\prime}(z)\right|=e^{-u}$. But the fact that $d u=0$ at $x_{0}^{\prime}$ clearly implies that $b=0$, and it follows that:

$$
\left|f^{\prime}(z)\right|^{2}=\left|1+\frac{c}{2} z^{2}+o\left(z^{2}\right)\right|^{2}=1+\operatorname{Re}\left(c z^{2}\right)+o\left(z^{2}\right)
$$

This means that:

$$
\operatorname{Re}\left(c z^{2}\right)=-\Pi_{0}^{*}(z, z)
$$

where $I_{0}^{*}$ is considered as a real-valued symmetric bilinear form on $\mathbb{C}$, identified with $T_{x_{0}^{\prime}} \partial_{i} M^{\prime}$. So

$$
\operatorname{Re}\left(\left(\phi_{0}^{-1} \circ \phi\right)^{\prime \prime \prime}\left(x_{0}^{\prime}\right)\right)=-I_{0}^{*}
$$

which means precisely that $2 \Pi_{0}^{*}=-R e(\mathcal{S}(\phi))$, as claimed.

\section{References}

[And01] Michael T. Anderson. $L^{2}$ curvature and volume renormalization of AHE metrics on 4-manifolds. Math. Res. Lett., 8(1-2):171-188, 2001.

[Ber60] Lipman Bers. Simultaneous uniformization. Bull. Amer. Math. Soc., 66:94-97, 1960.

[Bes87] A. Besse. Einstein Manifolds. Springer, 1987.

[BK99] Vijay Balasubramanian and Per Kraus. A stress tensor for anti-de Sitter gravity. Comm. Math. Phys., 208(2):413-428, 1999 .

[BO04] Francis Bonahon and Jean-Pierre Otal. Laminations mesurées de plissage des variétés hyperboliques de dimension 3. Ann. Math., 160:1013-1055, 2004.

[CS06] Young-Eun Choi and Caroline Series. Lengths are coordinates for convex structures. J. Differential Geom., 73(1):75-117, 2006.

[Eps84] Charles L. Epstein. Envelopes of horospheres and Weingarten surfaces in hyperbolic 3-space. Preprint, 1984.

[Hop51] Heinz Hopf. Über Flächen mit einer Relation zwischen den Hauptkrümmungen. Math. Nachr., 4:232$249,1951$.

[HS98] Mark Henningson and Kostas Skenderis. The holographic weyl anomaly. JHEP, 9807:023, 1998.

[Kra00] Kirill Krasnov. Holography and Riemann surfaces. Adv. Theor. Math. Phys., 4(4):929-979, 2000. 
[Kra01] Kirill Krasnov. Three-dimensional gravity, point particles and Liouville theory. Classical Quantum Gravity, 18(7):1291-1304, 2001.

[KS05] Kirill Krasnov and Jean-Marc Schlenker. Minimal surfaces and particles in 3-manifolds. math.DG/0511441, 2005.

[Lec06] Cyril Lecuire. Plissage des variétés hyperboliques de dimension 3. Invent. Math., 164(1):85-141, 2006.

[McM00] Curtis T. McMullen. The moduli space of Riemann surfaces is Kähler hyperbolic. Ann. of Math. (2), 151(1):327-357, 2000.

[MS06] Sergiu Moroianu and Jean-Marc Schlenker. Quasi-Fuchsian manifolds with particles. math.DG/060344, v2., 2006.

[OS98] Brad Osgood and Dennis Stowe. The Schwarzian derivative, conformal connections, and Möbius structures. J. Anal. Math., 76:163-190, 1998.

[PP01] S. J. Patterson and Peter A. Perry. The divisor of Selberg's zeta function for Kleinian groups. Duke Math. J., 106(2):321-390, 2001. Appendix A by Charles Epstein.

[RH93] Igor Rivin and Craig D. Hodgson. A characterization of compact convex polyhedra in hyperbolic 3-space. Invent. Math., 111:77-111, 1993.

[RS99] Igor Rivin and Jean-Marc Schlenker. The Schläfli formula in Einstein manifolds with boundary. Electronic Research Announcements of the A.M.S., 5:18-23, 1999.

[Sch02] Jean-Marc Schlenker. Hypersurfaces in $H^{n}$ and the space of its horospheres. Geom. Funct. Anal., 12:395-435, 2002.

[Teo05] Lee-Peng Teo. A different expression of the Weil-Petersson potential on the quasi-Fuchsian deformation space. Lett. Math. Phys., 73(2):91-107, 2005.

[Tro91] Marc Troyanov. Prescribing curvature on compact surfaces with conical singularities. Trans. Amer. Math. Soc., 324(2):793-821, 1991.

[TT03] Leon A. Takhtajan and Lee-Peng Teo. Liouville action and Weil-Petersson metric on deformation spaces, global Kleinian reciprocity and holography. Comm. Math. Phys., 239(1-2):183-240, 2003.

[TZ87] L. Takhtajan and P. Zograf. On uniformization of Riemann surfaces and the Weil-Petersson metric on the Teichmüller and Schottky spaces. Mat. Sb., 132:303-320, 1987. English translation in Math. USSR Sb. 60:297-313, 1988.

[Wit98] E. Witten. Anti de Sitter space and holography. Adv. Theor. Math. Phys., 2:253-291, 1998.

[Wit89] Edward Witten. 2+1-dimensional gravity as an exactly soluble system. Nuclear Phys. B, 311(1):46-78, 1988/89. 